Pacific

Journal of

Mathematics

CLASSIFICATION OF IRREDUCIBLE INTEGRABLE MODULES FOR TWISTED TOROIDAL LIE ALGEBRAS WITH FINITE-DIMENSIONAL WEIGHT SPACES

S. Eswara RAO AND PUnita Batra 


\title{
CLASSIFICATION OF IRREDUCIBLE INTEGRABLE MODULES FOR TWISTED TOROIDAL LIE ALGEBRAS WITH FINITE-DIMENSIONAL WEIGHT SPACES
}

\author{
S. ESWARA RAO AND PUnita Batra
}

\begin{abstract}
This paper classifies irreducible, integrable modules for "twisted toroidal Lie algebras" with finite-dimensional weight spaces. We prove that these modules turn out to be modules of appropriate direct sums of finitely many copies of affine Kac-Moody Lie algebras.
\end{abstract}

\section{Introduction}

Let $\mathfrak{g}$ be a finite-dimensional simple Lie algebra over the complex field $\mathbb{C}$. Let $A_{n}=\mathbb{C}\left[t_{1}^{ \pm 1}, \ldots, t_{n}^{ \pm 1}\right]$ be a Laurent polynomial ring in $n$ commuting variables $t_{1}, t_{2}, \ldots, t_{n}$. For $n>1$, we consider the multiloop Lie algebra $\mathfrak{g} \otimes \mathbb{C}\left[t_{1}^{ \pm 1}, \ldots, t_{n}^{ \pm 1}\right]$. The universal central extension of $\mathfrak{g} \otimes \mathbb{C}\left[t_{1}^{ \pm 1}, \ldots, t_{n}^{ \pm 1}\right]$ is called a toroidal Lie algebra. If $n=1$, then it is well known that the universal central extension of a loop algebra $\mathfrak{g} \otimes \mathbb{C}\left[t^{ \pm 1}\right]$ is one-dimensional. In this case, the universal central extension is an affine Kac-Moody Lie algebra. On the other hand, in the toroidal case, that is, when $n>1$, the universal central extension is infinite-dimensional. Toroidal Lie algebras are described in [Rao and Moody 1994]. Representations of toroidal Lie algebras have been studied in [Berman and Billig 1999] and [Rao 2004].

In Section 1, we define the twisted toroidal Lie algebra " $\tau(\mu)$ ", where $\mu$ is a Dynkin diagram automorphism of $\mathfrak{g}$ of order $m$. In the one variable case, $\tau(\mu)$ is just the twisted affine Kac-Moody Lie algebra [Kac 1990]. Rao [1993] gave some representations of the twisted affine Kac-Moody Lie algebra. Batra [2004] classified finite-dimensional irreducible representations of the twisted multiloop Lie algebras.

In this paper, we twist several variables at the same time, because twisting one variable restricts the class of twisted toroidal Lie algebras to one for each variable. By twisting several variables, we are covering a larger class of twisted toroidal Lie algebras. Now GL $(n, \mathbb{Z})$, the group of $n \times n$ matrices with entries in $\mathbb{Z}$, acts on $\mathbb{Z}^{n}$ naturally, and an element of $\operatorname{GL}(n, \mathbb{Z})$ defines an automorphism of the nontwisted toroidal Lie algebra " $\tau$ ". This automorphism is change of coordinates as described

MSC2000: 17B65.

Keywords: twisted toroidal Lie algebras, integrable modules. 
in Section 1. We consider $\tau(\mu)$ up to a suitable change of coordinates, and in this way, we can reduce the number of twisted variables to a minimum of two variables; see Remark 1.1.

Among all the modules of a toroidal Lie algebra, the so-called integrable modules (where the real root spaces act locally nilpotently) are very important since they are the ones that lift to representations of the corresponding group. Rao [2004] classified irreducible integrable modules for nontwisted toroidal Lie algebras with finite-dimensional weight spaces. Here we classify the irreducible integrable modules for twisted toroidal Lie algebras having finite-dimensional weight spaces, where the center acts nontrivially. Some of the ideas used in [Rao 2004] go through here, but this is a more complex case and needs new ideas.

In Section 1, we describe the root system for $\tau(\mu)$ and the Weyl group of $\tau(\mu)$, and we define integrable $\tau(\mu)$-modules. In Section 2, we define central operators on an irreducible module for $\tau(\mu)$. In this paper we classify those irreducible integrable $\tau(\mu)$-modules $V$ with finite-dimensional weight spaces in which some zero degree generators of the center of $\tau(\mu)$ act nontrivially. Then by change of coordinates, we assume that one of the zero degree generators of the center of $\tau(\mu)$, namely " $K_{n}$ ", acts nontrivially, while the others act trivially. Assuming that $K_{n}$ acts as a positive integer, we prove in Proposition 2.6 the existence of a highest weight vector in $V$.

In Section 3, we give the notion of a non-graded $\tau(\mu)$-module $V(\psi)$, define the graded $\tau(\mu)$-module $V(\psi)$, and, in Proposition 3.3, establish a one-to-one correspondence between non-graded and graded modules. So our problem reduces to classifying irreducible integrable non-graded $\tau(\mu)$-modules. In Section 4 , we prove that any such non-graded module is actually a module for $\left(\mathfrak{g}_{\text {aff }} \otimes\right.$ $\left.\mathbb{C}\left[t_{1}^{ \pm 1}, \ldots, t_{n-1}^{ \pm 1}\right]\right) \cap \tau(\mu)$, where $\mathfrak{g}_{\text {aff }}=\mathfrak{g} \otimes \mathbb{C}\left[t_{n}, t_{n}^{-1}\right] \oplus \mathbb{C} K_{n}$ is an affine Lie algebra. In Proposition 4.1, we give the conditions under which the Lie algebra homomorphism $\phi(\mu)$ from $\left(\mathfrak{g}_{\text {aff }} \otimes \mathbb{C}\left[t_{1}^{ \pm 1}, \ldots, t_{n-1}^{ \pm 1}\right]\right) \cap \tau(\mu)$ to $\bigoplus \mathfrak{g}_{\text {aff }}$ (the direct sum of finitely many copies of $\left.\mathfrak{g}_{\text {aff }}\right)$ becomes surjective. In Proposition 5.3, we prove that non-graded modules are zero on kernel of $\phi(\mu)$, and hence the nongraded modules actually become modules for $\bigoplus \mathfrak{g}_{\text {aff }}$. It is well known that the irreducible integrable modules of $\bigoplus \mathfrak{g}_{\text {aff }}$ are tensor products of irreducible highest weight modules [Rao 2005]. The main result of this paper is Theorem 3.4. Proved in Section 5, it says that any irreducible integrable module for $\tau(\mu)$ with finitedimensional weight spaces, on which the center acts nontrivially, is an irreducible component of $V(\psi) \otimes \mathbb{C}\left[t_{1}^{ \pm 1}, \ldots, t_{n-1}^{ \pm 1}\right]$.

We consider cases $m=2$ and $m=3$ separately. The modules for $\tau(\mu)$, where $\mu$ is a Dynkin diagram automorphism of order 3, are worked out in Section 6. 


\section{Preliminaries}

Let $\mathfrak{g}$ be a finite-dimensional simple Lie algebra of rank $d$ over the complex field $\mathbb{C}$. Let $\mathfrak{h}$ be a Cartan subalgebra of $\mathfrak{g}$. Fix a positive integer $n \geq 2$. Let $A=$ $A_{n}=\mathbb{C}\left[t_{1}^{ \pm 1}, \ldots, t_{n}^{ \pm 1}\right]$ be a Laurent polynomial ring in $n$ commuting variables $t_{1}, t_{2}, \ldots, t_{n}$. For $\underline{m}=\left(m_{1}, \ldots, m_{n}\right) \in \mathbb{Z}^{n}$, let $t^{\underline{m}}=t_{1}^{m_{1}} \cdots t_{n}^{m_{n}} \in A$. Then $\mathfrak{g}_{A}=\mathfrak{g} \otimes A$ can be made into a Lie algebra by defining

$$
\left[X \otimes t^{\underline{r}}, Y \otimes t^{\underline{s}}\right]=[X, Y] \otimes t^{\underline{r}+\underline{s}}
$$

for $X, Y \in \mathfrak{g}$ and $\underline{r}, \underline{s} \in \mathbb{Z}^{n}$. Suppose $\Omega_{A}$ is a complex vector space with basis $\left\{t \underline{m} K_{i} \mid 1 \leq i \leq n, \underline{m} \in \mathbb{Z}^{n}\right\}$, and suppose $d_{A}$ is the subspace of $\Omega_{A}$ spanned by $\left\{\sum m_{i} t^{\underline{m}} K_{i} \mid \underline{m} \in \mathbb{Z}^{n}\right\}$. Let $(\cdot, \cdot)$ be a nondegenerate, symmetric, $\mathfrak{g}$-invariant bilinear form on $\mathfrak{g}$. Let $\tau_{0}=\mathfrak{g} \otimes A \oplus \Omega_{A} / d_{A}$ be a Lie algebra defined by

- $\left[X \otimes t^{\underline{r}}, Y \otimes t^{\underline{s}}\right]=[X, Y] \otimes t^{\underline{r}+\underline{s}}+(X, Y) \sum r_{i} t^{\underline{r}+\underline{s}} K_{i}$ and

- $\Omega_{A} / d_{A}$ is central in $\tau_{0}$.

The Lie algebra $\tau_{0}$ is the universal central extension of $\mathfrak{g} \otimes A$ [Morita and Yoshii $1985]$ and is naturally $\mathbb{Z}^{n}$-graded. To reflect this fact, we consider a bigger Lie algebra. Let $\tau=\tau_{0} \oplus D$, where $D$ is the complex linear span of derivations $d_{1}, \ldots, d_{n}$. We now define a Lie algebra structure on $\tau$ by

$$
\begin{aligned}
{\left[d_{i}, X \otimes t^{\underline{r}}\right] } & =r_{i} X \otimes t^{\underline{\underline{r}}} & & \text { for } \underline{r} \in \mathbb{Z}^{n} \text { and } 1 \leq i \leq n, \\
{\left[d_{i}, t^{\underline{r}} K_{j}\right] } & =r_{i} t^{\underline{r}} K_{j} & & \text { for } \underline{r} \in \mathbb{Z}^{n} \text { and } 1 \leq i, j \leq n, \\
{\left[d_{i}, d_{j}\right] } & =0 & & \text { for } 1 \leq i, j \leq n .
\end{aligned}
$$

We call $\tau$ the nontwisted toroidal Lie algebra. We will now define the twisted toroidal Lie algebra. Let $\mu$ be a Dynkin diagram automorphism of $\mathfrak{g}$ of order $m$ and $\epsilon$ a primitive $m$-th root of unity. Let $S=\left\{i_{1}, i_{2}, \ldots, i_{k}\right\} \subset\{1,2, \ldots, n\}$ for $k>0$. Define an automorphism of $\tau$, again denoted by $\mu$, such that

$$
\begin{aligned}
\mu\left(X \otimes t^{\underline{r}}\right) & =(\epsilon)^{r_{i_{1}}+\cdots+r_{i_{k}}} \mu(X) \otimes t^{\underline{\underline{r}}}, \\
\mu\left(t^{\underline{r}} K_{i}\right) & =(\epsilon)^{r_{i_{1}}+\cdots+r_{i_{k}}} t^{\underline{r}} K_{i}, \\
\mu\left(d_{i}\right) & =d_{i} .
\end{aligned}
$$

It can be easily checked that $\mu$ is an automorphism of $\tau$ of order $m$. The subalgebra of $\mu$-fixed points of $\tau$ is called the twisted toroidal Lie algebra and is denoted by $\tau(\mu)$. Since $\mu$ leaves $\mathfrak{h}$ invariant, we let $\mathfrak{h}(\mu)$ be the $\mu$-fixed points of $\mathfrak{h}$ under $\mu$. Letting $m=2$, we have the decomposition $\mathfrak{g}=\mathfrak{g}_{0} \oplus \mathfrak{g}_{1}$ of $\mathfrak{g}$, where $\mathfrak{g}_{0}=\{X \in \mathfrak{g} \mid \mu(X)=X\}$ and $\mathfrak{g}_{1}=\{X \in \mathfrak{g} \mid \mu(X)=\epsilon X\}$. 
Suppose $(X, Y) \neq 0$ implies $X, Y \in \mathfrak{g}_{0}$ or $X, Y \in \mathfrak{g}_{1}$. Then the center of the twisted toroidal Lie algebra, which we denote by $\left(\Omega_{A} / d_{A}\right)^{0}$, will be

$$
\left(\Omega_{A} / d_{A}\right)^{0}=\operatorname{span}\left\{t^{\underline{m}} K_{i} \mid m_{i_{1}}+\cdots+m_{i_{k}} \cong 0(\bmod 2)\right\} .
$$

Also $\mu$ leaves $\mathfrak{g} \otimes A$ invariant. Let $(\mathfrak{g} \otimes A)_{\mu}$ be the $\mu$-fixed points of $\mathfrak{g} \otimes A$. Then

$$
\tau(\mu)=(\mathfrak{g} \otimes A)_{\mu} \oplus\left(\Omega_{A} / d_{A}\right)^{0} \oplus D .
$$

It is known from [Berman and Krylyuk 1995] that $(\mathfrak{g} \otimes A)_{\mu} \oplus\left(\Omega_{A} / d_{A}\right)^{0}$ is the universal central extension of $(\mathfrak{g} \otimes A)_{\mu}$. Let $\tau_{0}(\mu)=(\mathfrak{g} \otimes A)_{\mu} \oplus\left(\Omega_{A} / d_{A}\right)^{0}$.

Change of coordinates. We recall that $\mathrm{GL}(n, \mathbb{Z})$, the group of $n \times n$ matrices with entries in $\mathbb{Z}$ and determinant \pm 1 , acts naturally on $\mathbb{Z}^{n}$. Denote the action as $A \underline{m}$ for $A \in \mathrm{GL}(n, \mathbb{Z})$ and $\underline{m} \in \mathbb{Z}^{n}$. We define an automorphism on $\tau$ by

$$
\begin{aligned}
& A . X \otimes t^{\underline{m}}=X \otimes t^{A} \underline{\underline{m}}, \\
& A . d\left(t^{\underline{r}}\right) t^{\underline{s}}=d\left(t^{A \underline{r}}\right) t^{A \underline{s}}, \quad \text { where } d\left(t^{\underline{r}}\right) t^{\underline{s}}=\sum r_{i} t^{\underline{r}+\underline{s}} K_{i} .
\end{aligned}
$$

Let $\left(d_{1}^{1}, \ldots, d_{n}^{1}\right)=\left(A^{T}\right)^{-1}\left(d_{1}, \ldots, d_{n}\right)$. Then define $A\left(d_{i}\right)=d_{i}^{1}$ for $1 \leq i \leq n$. It is easy to check that $A$ is an automorphism on $\tau$. This automorphism is nothing but the change of coordinates. For example, set $s_{i}=t^{A} \underline{e_{i}}$, where $\left\{\underline{e_{1}}, \ldots, \underline{e_{n}}\right\}$ is the standard basis of $\mathbb{Z}^{n}$. Then clearly

$$
\mathbb{C}\left[s_{1}^{ \pm 1}, \ldots, s_{n}^{ \pm 1}\right]=\mathbb{C}\left[t_{1}^{ \pm 1}, \ldots, t_{n}^{ \pm 1}\right] .
$$

Remark 1.1. We will consider $\tau(\mu)$ up to a suitable change of coordinates. In this process, the set $S$ will be changed. In general, the set $S$ can be reduced to a minimum of two element set. Consider an example: Let $\mu$ be a Dynkin diagram automorphism of $\mathfrak{g}$ of order 2 . Let $S=\left\{i_{1}, i_{2}, \ldots, i_{k}\right\} \subset\{1,2, \ldots, n\}$. We assume that $k$ is odd. Put

$$
s_{1}=t_{i_{1}} t_{i_{2}} \cdots t_{i_{k}}, \quad s_{2}=t_{i_{1}}, \quad s_{3}=t_{i_{1}} t_{i_{2}}, \quad \ldots \quad, s_{k}=t_{i_{k-2}} t_{i_{k-1}} .
$$

For $i \in\{1,2, \ldots, n\}-S$, we put $s_{i}=t_{i}$. Then it is easy to see that

$$
\mathbb{C}\left[s_{1}^{ \pm 1}, \ldots, s_{k}^{ \pm 1}\right]=\mathbb{C}\left[t_{i_{1}}^{ \pm 1}, \ldots, t_{i_{k}}^{ \pm 1}\right] \quad \text { and } \quad \mathbb{C}\left[s_{1}^{ \pm 1}, \ldots, s_{n}^{ \pm 1}\right]=\mathbb{C}\left[t_{1}^{ \pm 1}, \ldots, t_{n}^{ \pm 1}\right] .
$$

Now the set $S$ changes with new variables $s_{1}, s_{2}, \ldots, s_{n}$, and $S=\{1,2\}$.

We note that $\tau(\mu)$ depends on the set $S$. But in this paper, we use a change of coordinates to reduce $S$ to $\{1, n\}$. So we are not including $S$ in the notation of $\tau(\mu)$.

Assume

$$
\tilde{\mathfrak{h}}=\mathfrak{h} \oplus \sum_{i=1}^{n} \mathbb{C} K_{i} \oplus \sum_{i=1}^{n} \mathbb{C} d_{i},
$$


which is a Cartan subalgebra of $\tau$. Consider $\mu$-fixed points $\tilde{\mathfrak{h}}(\mu)$ of $\tilde{\mathfrak{h}}$ under $\mu$. Then $\tilde{\mathfrak{h}}(\mu)=\mathfrak{h}(\mu) \oplus \sum_{i=1}^{n} \mathbb{C} K_{i} \oplus \sum_{i=1}^{n} \mathbb{C} d_{i}$ is a Cartan subalgebra of $\tau(\mu)$. It is well known that $\mathfrak{g}_{0}$, the fixed point subalgebra of $\mathfrak{g}$ under $\mu$, is a simple Lie algebra [Kac 1990], and $\mathfrak{h}(\mu)$ is a Cartan subalgebra of $\mathfrak{g}_{0}$.

Let $\alpha_{1}, \alpha_{2}, \ldots, \alpha_{l} \in \mathfrak{h}(\mu)^{*}$ be the simple roots of $\mathfrak{g}_{0}$. For $1 \leq i \leq l$, we extend the $\alpha_{i}$ to $\tilde{\mathfrak{h}}(\mu)^{*}$ by defining $\alpha_{i}\left(K_{j}\right)=\alpha_{i}\left(d_{j}\right)=0$ for $1 \leq j \leq n$. Let $\alpha_{1}^{\vee}, \ldots, \alpha_{l}^{\vee} \in \mathfrak{h}(\mu)$ be the coroots of $\mathfrak{g}_{0}$. For $1 \leq i \leq n$, define $\delta_{i} \in \tilde{\mathfrak{h}}(\mu)^{*}$ and $w_{i} \in \tilde{\mathfrak{h}}(\mu)^{*}$ by

$$
\begin{aligned}
\delta_{i}\left(\alpha_{j}^{\vee}\right)=0, & w_{i}\left(\alpha_{j}^{\vee}\right)=0 & & \text { for } 1 \leq j \leq l, \\
\delta_{i}\left(K_{j}\right)=0, & w_{i}\left(K_{j}\right)=\delta_{i j} & & \text { for } 1 \leq j \leq n, \\
\delta_{i}\left(d_{j}\right)=\delta_{i j}, & w_{i}\left(d_{j}\right)=0 & & \text { for } 1 \leq j \leq n,
\end{aligned}
$$

Then it is easy to see that $\alpha_{1}, \alpha_{2}, \ldots, \alpha_{l}, \delta_{1}, \ldots, \delta_{n}, w_{1}, \ldots, w_{n}$ is a $\mathbb{C}$-basis of $\tilde{\mathfrak{h}}(\mu)^{*}$.

Recall that the nondegenerate, symmetric, $\mathfrak{g}$-invariant bilinear form $(\cdot, \cdot)$ remains nondegenerate on $\mathfrak{g}_{0}$ as well as on $\mathfrak{h}(\mu)$. The form $(\cdot, \cdot)$ induces a nondegenerate form $\langle\cdot, \cdot\rangle$ on $\tilde{\mathfrak{h}}(\mu)^{*}$ defined as

$$
\begin{aligned}
\left\langle\alpha_{i}, \alpha_{j}\right\rangle & =\left(\alpha_{i}, \alpha_{j}\right) & & \text { for } 1 \leq i, j \leq l, \\
\left\langle\alpha_{i}, \delta_{j}\right\rangle & =0=\left\langle\alpha_{i}, w_{j}\right\rangle & & \text { for } 1 \leq i \leq l \text { and } 1 \leq j \leq n, \\
\left\langle\delta_{i}, \delta_{j}\right\rangle & =0=\left\langle w_{i}, w_{j}\right\rangle, & & \\
\left\langle\delta_{i}, w_{j}\right\rangle & =\delta_{i j} & & \text { for } 1 \leq i, j \leq n .
\end{aligned}
$$

For $\underline{m}=\left(m_{1}, \ldots, m_{n}\right) \in \mathbb{Z}^{n}$, define $\delta_{\underline{m}}=\sum m_{i} \delta_{i}$ and note that $\left\langle\delta_{\underline{m}}, \delta_{\underline{m}}\right\rangle=0$.

Root system for $\tau(\mu)$. Let $m=2$. Then

$$
\tau(\mu)=\mathfrak{g}_{0} \otimes \sum_{m_{i_{1}}+\cdots+m_{i_{k}} \cong 0} \mathbb{C}\left(t^{\underline{m}} \oplus \mathfrak{g}_{1} \otimes \sum_{m_{i_{1}}+\cdots+m_{i_{k}} \cong 1(\bmod 2)} \mathbb{C} t^{\underline{m}} \oplus\left(\Omega_{A} / d_{A}\right)^{0} \oplus D .\right.
$$

Let $\Delta_{0}$ be the roots of $\mathfrak{g}_{0}$ and $\Delta_{0}^{s}$ be the short roots of $\mathfrak{g}_{0}$. Notice that the nonzero weights of the $\mathfrak{g}_{0}$-module $\mathfrak{g}_{1}$ are all short roots of $\mathfrak{g}_{0}$ except in $A_{2 l}^{(2)}$.

Define

$\Delta_{\mathrm{re}}=\left\{\alpha+\delta_{\underline{m}} \mid \alpha \in \Delta_{0}, m_{i_{1}}+\cdots+m_{i_{k}} \cong 0(\bmod 2)\right\}$

$$
\bigcup\left\{\alpha+\delta_{\underline{m}} \mid \alpha \in \Delta_{0}^{s}, m_{i_{1}}+\cdots+m_{i_{k}} \cong 1(\bmod 2)\right\}
$$

and $\Delta_{\text {im }}=\left\{\delta_{\underline{m}} \mid \underline{m} \in \mathbb{Z}^{n}\right\}$. Let $\Delta=\Delta_{\mathrm{re}} \cup \Delta_{\text {im }}$. For $\alpha \in \Delta$, let

$$
\tau_{\alpha}=\{x \in \tau(\mu) \mid[h, x]=\alpha(h) x \text { for all } h \in \tilde{\mathfrak{h}}(\mu)\} .
$$

Then clearly $\tau(\mu)=\bigoplus_{\alpha \in \Delta} \tau_{\alpha}$ is the root space decomposition of $\tau(\mu)$ with respect to $\tilde{\mathfrak{h}}(\mu)$. 
Define

$$
\begin{aligned}
\Delta_{\mathrm{re}}^{+} & =\left\{\alpha+\delta_{\underline{m}} \mid \alpha>0, m_{n} \geq 0 \text { or } \alpha<0, m_{n} \geq 1\right\}, \quad \Delta_{\mathrm{im}}^{+}=\left\{\delta_{\underline{m}} \mid m_{n} \geq 1\right\}, \\
\Delta^{0} & =\left\{\delta_{\underline{m}} \mid m_{n}=0\right\} .
\end{aligned}
$$

Then $\Delta^{+}=\Delta_{\mathrm{re}}^{+} \cup \Delta_{\text {im }}^{+}$and $\Delta^{-}=-\Delta^{+}$. Further $\Delta=\Delta^{+} \cup \Delta^{0} \cup \Delta^{-}$. Let $\alpha \in \Delta$. Then $\alpha \in \Delta_{\text {re }}$ if and only if $\langle\alpha, \alpha\rangle \neq 0$.

Coroots. Let $\alpha \in \Delta_{0}$. Then

$$
\alpha^{\vee}=\sum_{i=1}^{l} n_{i}\left(\left|\alpha_{i}\right|^{2} /|\alpha|^{2}\right) \alpha_{i}^{\vee} \text { for } \alpha=\sum_{i=1}^{l} n_{i} \alpha_{i} .
$$

It is easy to check that $\alpha\left(\alpha^{\vee}\right)=2$. Suppose $\alpha \in \Delta_{\text {re }}$ and $\alpha=\beta+\delta_{\underline{m}}$. Then define

$$
\alpha^{\vee}=\beta^{\vee}+\left(2 /|\beta|^{2}\right) \sum m_{i} K_{i} .
$$

Weyl group of $\boldsymbol{\tau}(\boldsymbol{\mu})$. For $\alpha \in \Delta_{\mathrm{re}}$, we define a reflection

$$
r_{\alpha}: \tilde{\mathfrak{h}}(\mu)^{*} \rightarrow \tilde{\mathfrak{h}}(\mu)^{*}, \quad \lambda \mapsto \lambda-\lambda\left(\alpha^{\vee}\right) \alpha .
$$

Let $W$ be the group generated by $r_{\alpha}, \alpha \in \Delta_{\text {re }}$. Then it is easy to prove that $\langle w \lambda, w \mu\rangle=\langle\lambda, \mu\rangle$ for all $w \in W$. W is called the Weyl group of $\tau(\mu)$.

Definition 1.2. A module $V$ of $\tau(\mu)$ is said to be integrable if

- $V=\bigoplus_{\lambda \in \tilde{\mathfrak{h}}(\mu)^{*}} V_{\lambda}$, where $V_{\lambda}=\{v \in V \mid h v=\lambda(h) v$ for all $h \in \tilde{\mathfrak{h}}(\mu)\}$;

- For all $\alpha+\delta_{\underline{m}} \in \Delta_{\text {re }}$ and $v \in V$, there exists an integer $k=k(\alpha, \underline{m}, v)$ such that $\left(X_{\alpha} \otimes t^{\underline{m}}\right)^{k} v=0$, where $X_{\alpha}$ is the root vector corresponding to root $\alpha$.

Lemma 1.3. Suppose $V$ is an irreducible integrable module for $\tau(\mu)$ with finitedimensional weight spaces. Let $P(V)$ denote the set of all weights of $V$. Then

(i) $P(V)$ is $W$-invariant;

(ii) $\operatorname{dim} V_{\lambda}=\operatorname{dim} V_{w \lambda}$ for $w \in W$ and $\lambda \in P(V)$;

(iii) if $\alpha \in \Delta_{\mathrm{re}}$ and $\lambda \in P(V)$, then $\lambda\left(\alpha^{\vee}\right) \in \mathbb{Z}$;

(iv) if $\alpha \in \Delta_{\text {re }}$ and $\lambda \in P(V)$, and if $\lambda\left(\alpha^{\vee}\right)>0$, then $\lambda-\alpha \in P(V)$.

(v) $\lambda\left(K_{i}\right)$ is a constant integer for all $\lambda \in P(V)$, for $i=1,2, \ldots, n$.

Proof. The proof is standard.

From now on we will consider $\tau(\mu)$ up to a change of coordinates. Recall that $\mu$ leaves the algebra $B=\mathbb{C}\left[t_{1}^{ \pm 1}, \ldots, t_{n-1}^{ \pm 1}\right]$ invariant. Let $B_{\mu}$ be the $\mu$-fixed points of $B$. Now $B$ can be seen as a group algebra of $\mathbb{Z}^{n-1}$. Similarly, $B_{\mu}$ can be seen as a group algebra of subgroup $\Gamma$ of $\mathbb{Z}^{n-1}$ of index 2. But by Remark 1.1, one can change the basis so that $\Gamma=2 \underline{e_{1}} \mathbb{Z} \oplus \underline{e_{2}} \mathbb{Z} \oplus \cdots \oplus \underline{e_{n-1}} \mathbb{Z}$. Thus we conclude that it 
is possible to change coordinates so that $S=\{1, n\}$. In this case when $m=2$, we have for $X \in \mathfrak{g}$ that

$$
\begin{aligned}
\mu\left(X \otimes t_{1}^{2 m_{1}} t_{2}^{m_{2}} \cdots t_{n-1}^{m_{n-1}} t_{n}^{2 m_{n}}\right) & =\mu(X) \otimes t_{1}^{2 m_{1}} t_{2}^{m_{2}} \cdots t_{n-1}^{m_{n-1}} t_{n}^{2 m_{n}}, \\
\mu\left(X \otimes t_{1}^{2 m_{1}+1} t_{2}^{m_{2}} \cdots t_{n-1}^{m_{n-1}} t_{n}^{2 m_{n}}\right) & =-\mu(X) \otimes t_{1}^{2 m_{1}+1} t_{2}^{m_{2}} \cdots t_{n-1}^{m_{n-1}} t_{n}^{2 m_{n}}, \\
\mu\left(X \otimes t_{1}^{2 m_{1}} t_{2}^{m_{2}} \cdots t_{n-1}^{m_{n-1}} t_{n}^{2 m_{n}+1}\right) & =-\mu(X) \otimes t_{1}^{2 m_{1}} t_{2}^{m_{2}} \cdots t_{n-1}^{m_{n-1}} t_{n}^{2 m_{n}+1}, \\
\mu\left(X \otimes t_{1}^{2 m_{1}+1} t_{2}^{m_{2}} \cdots t_{n-1}^{m_{n-1}} t_{n}^{2 m_{n}+1}\right) & =\mu(X) \otimes t_{1}^{2 m_{1}+1} t_{2}^{m_{2}} \cdots t_{n-1}^{m_{n-1}} t_{n}^{2 m_{n}+1} .
\end{aligned}
$$

So

$$
\begin{aligned}
\tau(\mu)=\bigoplus_{m \in \mathbb{Z}} \mathfrak{g}_{0} \otimes t_{n}^{2 m} B_{\mu} \bigoplus_{m \in \mathbb{Z}} \mathfrak{g}_{0} \otimes t_{n}^{2 m+1} t_{1} B_{\mu} \\
\bigoplus_{m \in \mathbb{Z}} \mathfrak{g}_{1} \otimes t_{n}^{2 m} t_{1} B_{\mu} \bigoplus_{m \in \mathbb{Z}} \mathfrak{g}_{1} \otimes t_{n}^{2 m+1} B_{\mu} \bigoplus\left(\Omega_{A} / d_{A}\right)^{0} \bigoplus D,
\end{aligned}
$$

where $B_{\mu}=\mathbb{C}\left[t_{1}^{ \pm 2}, t_{2}^{ \pm 1}, \ldots, t_{n-1}^{ \pm 1}\right]$.

Let $Q$ be the $\mathbb{Z}$-lattice spanned by $\alpha_{1}, \ldots, \alpha_{l}, \delta_{n}$. Recall $\mathfrak{g}_{0} \otimes \mathbb{C}\left[t_{n}, t_{n}^{-1}\right] \oplus \mathbb{C} K_{n}$ is an affine Lie algebra, which need not be a subalgebra of $\tau(\mu)$. Let $\beta$ be the highest root of the finite root system $\Delta_{0}$. Let $\alpha_{0}=-\beta+\delta_{n}$. Then it is well known that $\left\{\alpha_{1}, \ldots, \alpha_{l}, \alpha_{0}\right\}$ forms a system of simple roots for $\mathfrak{g}_{0} \otimes \mathbb{C}\left[t_{n}, t_{n}^{-1}\right] \oplus \mathbb{C} K_{n}$.

Let $Q(\mu)$ be the root lattice of $\tau(\mu)$. This is a $\mathbb{Z}$-lattice spanned by $\alpha_{1}, \ldots, \alpha_{l}$, $\delta_{1}, \ldots, \delta_{n}$. For $\lambda, \mu \in Q(\mu)$, we say $\lambda \geq \mu$ if

$$
\lambda-\mu=\sum_{i=0}^{l} n_{i} \alpha_{i} \quad \text { for } 0 \leq n_{i} \in \mathbb{Z} .
$$

Hence $\lambda>0$ means $\lambda \geq 0$ and $\lambda \neq 0$. Now consider the twisted affine Lie algebra, $\mathfrak{g}_{0} \otimes \mathbb{C}\left[t_{n}^{2}, t_{n}^{-2}\right] \oplus \mathfrak{g}_{1} \otimes t_{n} \mathbb{C}\left[t_{n}^{2}, t_{n}^{-2}\right] \oplus \mathbb{C} K_{n}$, which is also a subalgebra of $\tau(\mu)$. Its positive roots are also positive in the above sense.

\section{Central operators}

The purpose of this paper is to classify irreducible integrable modules for $\tau(\mu)$ with finite-dimensional weight spaces with respect to the Cartan subalgebra $\tilde{\mathfrak{h}}(\mu)$, where the center acts nontrivially. We first get information on a (fixed) irreducible module $V$ for $\tau(\mu)$ with finite-dimensional weight spaces.

Definition 2.1. A linear map $Z: V \rightarrow V$ is called a central operator of degree $\underline{m} \in \mathbb{Z}^{n}$ if $Z$ commutes with the $\tau_{0}(\mu)$ action and

$$
d_{i} Z-Z d_{i}=m_{i} Z \text { for all } i \text {. }
$$

For example, $t^{\underline{m}} K_{i}$ is a central operator of degree $\underline{m}$ and $t^{\underline{r}} K_{i} t^{\underline{s}} K_{j}$ is a central operator of degree $\underline{r}+\underline{s}$. 
Lemma 2.2. $\quad$ Let $Z$ be a central operator of degree $\underline{m}$ such that $Z v \neq 0$ for some $v \in V$. Then $Z w \neq 0$ for every nonzero $w \in V$.

- Let $Z$ be a nonzero central operator of degree $m$. Then there exists a central operator $T$ on $V$ of degree $-\underline{m}$ such that $Z T=T Z=\mathrm{Id}$.

- Let $Z_{1}$ and $Z_{2}$ be nonzero central operators of degree $m$ on $V$. Then there exists a scalar $\lambda$ such that $Z_{1}=\lambda Z_{2}$.

Proof. The proofs are easy and given in [Rao 2004].

Theorem 2.3. Let $V$ be an irreducible module for $\tau(\mu)$ with finite-dimensional weight spaces with respect to the Cartan subalgebra $\tilde{\mathfrak{h}}(\mu)$. Let

$$
L=\left\{\underline{m} \in Z^{n} \mid t^{\underline{m}} K_{i} \neq 0 \text { on } V \text { for some } i\right\} .
$$

Let $\langle L\rangle$ be the subgroup of $\mathbb{Z}^{n}$ generated by L. Suppose $\operatorname{rank}\langle L\rangle=k \leq n$. Then up to a coordinate change,

(i) there exist nonzero positive integers $k_{1}, \ldots, k_{k}$ and nonzero central operators $Z_{1}, Z_{2}, \ldots, Z_{k}$ of degrees $\left(k_{1}, 0, \ldots, 0\right), \ldots,\left(0, \ldots, k_{k}, 0, \ldots, 0\right)$, respectively;

(ii) $k<n$;

(iii) $t^{\underline{r}} K_{i} \neq 0$ on $V$ implies that $i \geq k+1$ and $r_{k+1}=\cdots=r_{n}=0$;

(iv) there exists a proper submodule $W$ of $\tau_{0}(\mu) \oplus D_{k}$, where $D_{k}$ is the linear span of $\left\{d_{k+1}, \ldots, d_{n}\right\}$, such that $V / W$ has finite-dimensional weight spaces with respect to $\mathfrak{h}(\mu) \bigoplus \sum_{i=1}^{n} \mathbb{C} K_{i} \bigoplus D_{k}$.

Proof. The proofs are exactly the same as those of [Rao 2004, Theorem 4.5].

Theorem 2.4. With the notation above, suppose there is an element in $\left(\Omega_{A} / d_{A}\right)^{0}$ that acts nontrivially on $V$. Then $k=n-1$.

Proof. The proof is the same as that of [Rao 2004, Proposition 4.8].

Proposition 2.5. Let $\bar{\tau}(\mu)$ be the quotient of $\tau(\mu)$ by all nonzero-degree central operators. Suppose $\bar{V}$ is a module for $\bar{\tau}(\mu)$ with finite-dimensional weight spaces. Then the zero-degree central operators $K_{i}$ for $1 \leq i \leq n$ act trivially on $\bar{V}$.

Proof. The proof follows from [Rao 2004].

Let $V$ be an irreducible integrable module for $\tau(\mu)$ with finite-dimensional weight space. Choose coordinates using Theorems 2.3 and 2.4 , so that $t^{\underline{m}} K_{i} \neq 0$ on $V$ implies $m_{n}=0$ and $i=n$.

Proposition 2.6. Let $V$ be as above. Suppose $K_{n}$ acts as positive integer. Then there exists $\lambda \in P(V)$ such that $\lambda+\eta+\delta_{\underline{m}^{\prime}} \notin P(V)$ for all $\eta>0$, where $\delta_{\underline{m}^{\prime}}=$ $\sum_{i=1}^{n-1} m_{i} \delta_{i}$, and for all $\underline{m}^{\prime}=\left(m_{1}, m_{2}, \ldots, \bar{m}_{n-1}\right)$. 
Proof. The proof uses lemmas from [Rao 2004]; these we enclose in brackets.

Notice that $\mathfrak{g}_{0, \text { aff }}=\mathfrak{g}_{0} \otimes \mathbb{C}\left[t_{n}^{2}, t_{n}^{-2}\right] \oplus \mathbb{C} K_{n}$ is an affine subalgebra of $\tau(\mu)$. Then

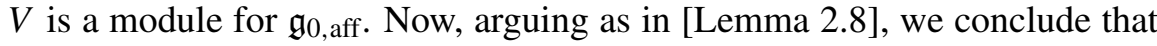

$$
\text { there exists a } \lambda \in P(V) \text { such that } \lambda+\eta \notin P(V) \text { for all } \eta>0 \text {. }
$$

Next we prove that

$$
\lambda+\eta+\delta_{1} \notin P(V) \text { for all } \eta>0 \text { or } \lambda+\eta-\delta_{1} \notin P(V) \text { for all } \eta>0 .
$$

For proving $(* *)$, first we get $\lambda \in P(V)$ such that $\lambda+\eta \notin P(V)$ for all $\eta>0$. Suppose $\lambda+\eta+\delta_{1} \in P(V)$ for some $\eta>0$. Then, arguing as in [Lemma 2.8], we get $\eta^{\prime} \geq 0$ such that $\lambda+\eta+\delta_{1}+\eta^{\prime} \in P(V)$ and $\lambda+\eta+\delta_{1}+\eta^{\prime}+\eta^{\prime \prime} \notin P(V)$ for all $\eta^{\prime \prime}>0$. Also for all $\eta^{\prime \prime}>0, \lambda+\eta+\delta_{1}+\eta^{\prime}+\eta^{\prime \prime}-\delta_{1}=\lambda+\eta+\eta^{\prime}+\eta^{\prime \prime} \notin P(V)$, since $\eta+\eta^{\prime}+\eta^{\prime \prime}>0$.

Case 2.6a. $m=2$.

Suppose that there exists a $\lambda \in P(V)$ such that $\lambda+\eta \notin P(V)$ and $\lambda+\eta+\delta_{1} \notin P(V)$ for all $\eta>0$. If $\lambda+\alpha+\delta_{m} \notin P(V)$ for all $\alpha+\delta_{m} \in \Delta_{\text {re }}^{+}$, then we are done. Suppose $\lambda+\alpha+\delta_{\underline{m}} \in P(V)$ for some $\alpha+\delta_{\underline{m}} \in \Delta_{\mathrm{re}}^{+}$. Let $\mu=\lambda+\alpha+\delta_{\underline{m}}$.

We claim $\mu+\beta+\delta_{\underline{s}} \notin P(V)$ for all $\beta+\delta_{\underline{s}} \in \Delta_{\mathrm{re}}^{+}$. Supposing it is false, let $\mu+\beta+\delta_{\underline{s}} \in P(V)$ for some $\beta+\delta_{\underline{s}} \in \Delta_{\mathrm{re}}^{+}$.

Case 2.6a1. $\left\langle\alpha+\beta+\delta_{m}+\delta_{s}, \alpha+\delta_{m}\right\rangle>0$.

Let $\alpha=\alpha^{\prime}+\delta_{\underline{m}^{\prime}}$, where $\delta_{\underline{m}^{\prime}}=\sum_{i=1}^{n-1} m_{i} \delta_{i}$, and $\beta=\beta^{\prime}+\delta_{\underline{s^{\prime}}}$, where $\delta_{\underline{s}^{\prime}}=\sum_{i=1}^{n-1} s_{i} \delta_{i}$, where $\alpha^{\prime}, \beta^{\prime}>0$ and $\alpha^{\prime}, \beta^{\prime} \in \mathfrak{g}_{0, \text { aff. }}$.

Suppose $\alpha+\delta_{\underline{s}^{\prime}} \in \Delta_{\mathrm{re}}$. Then by [Lemma 1.4(2)] we have $\lambda+\alpha+\beta+\delta_{\underline{m}}+$ $\delta_{\underline{\underline{s}}}\left(\left(\alpha+\delta_{\underline{s}^{\prime}}\right)^{\vee}\right)>0$, since $\lambda\left(\alpha+\delta_{\underline{s^{\prime}}}\right)^{\vee}>0$.

By Lemma 1.3(iv), $\lambda+\alpha+\beta+\delta_{\underline{m}}+\delta_{\underline{s}}-\left(\alpha+\delta_{\underline{s}^{\prime}}\right) \in P(V)$. This implies $\lambda+\beta^{\prime}+\delta_{\underline{m}}+\delta_{\underline{s}} \in P(V)$. We note that $\beta^{\prime}+\delta_{\underline{m}}+\delta_{\underline{s}}>0$. Since $\alpha+\delta_{\underline{m}}$ and $\beta+\delta_{\underline{s}}$ are in $\Delta_{\text {re }}^{+}$, the $n$-th component $m_{n}$ of $\underline{m}$ and the $n$-th component $s_{n}$ of $\underline{s}$ are $\geq 0$. Hence $\beta^{\prime}+\delta_{\underline{m}}+\delta_{\underline{s}}>0$. So $\lambda+\beta^{\prime}+\delta_{\underline{m}}+\delta_{\underline{s}} \in P(V)$, which contradicts $(*)$.

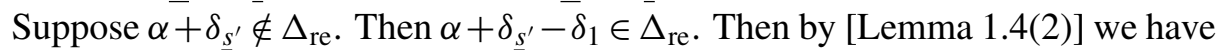
$\lambda+\alpha+\beta+\delta_{\underline{m}}+\delta_{\underline{s}}\left(\left(\alpha+\delta_{\underline{s}^{\prime}}-\delta_{1}\right)^{\vee}\right)>0$. Notice that $\lambda\left(\delta_{i}\right)=0$ for every $1 \leq i \leq n-1$. Thus by Lemma 1.3(iv), $\lambda+\alpha+\beta+\delta_{\underline{m}}+\delta_{\underline{s}}-\alpha-\delta_{\underline{\underline{s}^{\prime}}}+\delta_{1}=\lambda+\beta^{\prime}+\delta_{\underline{m}}+\delta_{\underline{s}}+\delta_{1} \in$ $P(V)$. Again as above $\beta^{\prime}+\delta_{\underline{m}}+\delta_{\underline{s}}>0$. So $\lambda+\beta^{\prime}+\delta_{\underline{m}}+\delta_{\underline{s}}+\delta_{1} \in P(V)$, which contradicts $(* *)$.

Case 2.6a2. $\left\langle\alpha+\beta+\delta_{\underline{m}}+\delta_{\underline{s}}, \beta+\delta_{\underline{s}}\right\rangle>0$.

This can be done as in Case 2.6a1.

Case 2.6a3. $\left\langle\alpha+\beta+\delta_{\underline{m}}+\delta_{\underline{s}}, \alpha+\beta+\delta_{\underline{m}}+\delta_{\underline{s}}\right\rangle \geq 0$.

Note that $\left\langle\alpha+\beta+\delta_{\underline{m}}+\delta_{\underline{s}}, \alpha+\beta+\delta_{\underline{m}}+\delta_{\underline{s}}\right\rangle=\left\langle\alpha^{\prime}+\beta^{\prime}, \alpha^{\prime}+\beta^{\prime}\right\rangle$. Thus the cases $\left\langle\alpha^{\prime}+\beta^{\prime}, \alpha^{\prime}\right\rangle>0$ and $\left\langle\alpha^{\prime}+\beta^{\prime}, \beta^{\prime}\right\rangle>0$ are already done. Note that $\alpha^{\prime}, \beta^{\prime}$ are in affine root system and $\langle\cdot, \cdot\rangle$ is a semipositive definite form in the affine root lattice. 
So we are left with the case $\left\langle\alpha^{\prime}+\beta^{\prime}, \alpha^{\prime}+\beta^{\prime}\right\rangle=0$. Thus by standard arguments, $\alpha^{\prime}+\beta^{\prime}=l \delta_{n}$ for some $l>0$, and further $\left\langle\alpha^{\prime}+\beta^{\prime}, \alpha^{\prime}\right\rangle=\left\langle\alpha^{\prime}+\beta^{\prime}, \beta^{\prime}\right\rangle=0$. Now either $\alpha^{\prime}$ or $\alpha^{\prime}+\delta_{1} \in \Delta_{\mathrm{re}}^{+}$. In any case, $\lambda\left(\alpha^{\prime \vee}\right) \geq 0$. (Since $\lambda+\alpha^{\prime}, \lambda+\alpha^{\prime}+\delta_{1} \notin P(V)$, by $(*)$ and $(* *)$.)

Suppose $\lambda\left(\alpha^{\prime \vee}\right)>0$.

Subcase 1. Suppose $\alpha+\delta_{\underline{s}^{\prime}} \in \Delta_{\text {re }}$. Then by [Lemma 1.4(2)] we have $\lambda+\alpha+\beta+$ $\delta_{\underline{m}}+\delta_{\underline{s}}\left(\left(\alpha+\delta_{\underline{s}^{\prime}}\right)^{\vee}\right)>0$, since $\left\langle\alpha+\beta+\delta_{\underline{m}}+\delta_{\underline{s}}, \alpha+\delta_{\underline{s}^{\prime}}\right\rangle=\left\langle\alpha^{\prime}+\beta^{\prime}, \alpha^{\prime}\right\rangle=0$. By Lemma 1.3(iv), $\lambda+\alpha+\beta+\delta_{\underline{m}}+\delta_{\underline{s}}-\alpha-\delta_{\underline{s}^{\prime}}=\lambda+\beta^{\prime}+\delta_{\underline{m}}+\delta_{\underline{s}} \in P(V)$, which contradicts $(*)$ since $\beta^{\prime}+\delta_{\underline{m}}+\delta_{\underline{s}}>0$.

Subcase 2. Suppose $\alpha+\delta_{\underline{s}^{\prime}}-\delta_{1} \in \Delta_{\text {re }}$. Then by [Lemma 1.4(2)] we have $\lambda+\alpha+\beta+$ $\delta_{\underline{m}}+\delta_{\underline{s}}\left(\left(\alpha+\delta_{\underline{s}^{\prime}}-\delta_{1}\right)^{\vee}\right)>0$. By Lemma 1.3(iv), $\lambda+\alpha+\beta+\delta_{\underline{m}}+\delta_{\underline{s}}-\alpha-\delta_{\underline{s}^{\prime}}+\delta_{1}=$ $\lambda+\beta^{\prime}+\delta_{\underline{m}}+\delta_{\underline{s}}+\delta_{1} \in P(V)$, which contradicts $(* *)$ since $\bar{\beta}^{\prime}+\bar{\delta}_{\underline{m}}+\delta_{\underline{s}}>0$.

Suppose $\lambda\left(\alpha^{\prime \vee}\right)=0$.

Subcase 1. Suppose $\alpha+\delta_{\underline{s}^{\prime}} \in \Delta_{\text {re. Then }} \lambda\left(\left(-\beta^{\prime}+l \delta_{n}+\delta_{\underline{m}^{\prime}}+\delta_{\underline{s}^{\prime}}\right)^{\vee}\right)=l \lambda\left(\delta_{n}\right)>0$ since $l>0$ and $\lambda\left(\delta_{n}\right)=K_{n}>0$. Now $\lambda+\alpha+\beta+\delta_{\underline{m}}+\delta_{\underline{s}}\left(\left(-\overline{\beta^{\prime}}+l \delta_{n}+\delta_{\underline{m}^{\prime}}+\delta_{\underline{s}^{\prime}}\right)^{\vee}\right)>0$. So by Lemma 1.3(iv), $\lambda+\alpha+\beta+\delta_{\underline{m}}+\delta_{\underline{s}}-\alpha^{\prime}-\delta_{\underline{m}^{\prime}}-\delta_{\underline{s}^{\prime}}=\lambda+\beta^{\prime}+\delta_{\underline{m}}+\delta_{\underline{s}} \in P(V)$, which contradicts $(*)$ since $\beta^{\prime}+\delta_{\underline{m}}+\delta_{\underline{s}}>0$.

Subcase 2. $\alpha+\delta_{\underline{s}^{\prime}}-\delta_{1} \in \Delta_{\text {re }}$ can be handled similarly.

This concludes the proof of Proposition 2.6.

\section{Classification of integrable $\tau(\mu)$-modules}

Let $\mathfrak{g}_{\text {aff }}=\mathfrak{g} \otimes \mathbb{C}\left[t_{n}, t_{n}^{-1}\right] \oplus \mathbb{C} K_{n}$. Consider $\mathfrak{g}_{\text {aff }} \otimes \mathbb{C}\left[t_{1}^{ \pm 1}, \ldots, t_{n-1}^{ \pm 1}\right] \oplus D$ and consider the subalgebra $\left(\mathfrak{h} \oplus \mathbb{C} K_{n}\right) \otimes \mathbb{C}\left[t_{1}^{ \pm 1}, \ldots, t_{n-1}^{ \pm 1}\right]$. Let

$$
H=\tau(\mu) \cap\left(\left(\mathfrak{h} \oplus \mathbb{C} K_{n}\right) \otimes \mathbb{C}\left[t_{1}^{ \pm 1}, \ldots, t_{n-1}^{ \pm 1}\right]\right) .
$$

Let $\tilde{H}=H \oplus D$. We have proved in Proposition 2.6 that there exists a $\lambda \in P(V)$ such that $V_{\lambda}$ is a "highest weight" space. In fact it is easy to see that $\bigoplus_{m^{\prime}} V_{\lambda+\delta_{m^{\prime}}}$ is also a highest weight space. Let $\bigoplus_{\underline{m}^{\prime}} V_{\lambda+\delta_{m^{\prime}}}=V^{\lambda}$. Note that $H$ is a graded abelian Lie algebra and $V^{\lambda}$ is a graded $H$-module. Since $V$ is irreducible, it follows by weight arguments that $V^{\lambda}$ is a graded irreducible $H$ module. From known facts [Rao 2004], it follows that $V^{\lambda}$ is isomorphic to a subalgebra $E$ of $\mathbb{C}\left[t_{1}^{ \pm 1}, \ldots, t_{n-1}^{ \pm 1}\right]$ such that each nonzero homogeneous element of $E$ is invertible. Then the $H$ module action on $V^{\lambda}$ can be described by a $\mathbb{Z}^{n-1}$ graded map from $H$ to $E$, which we call $\Psi$. Now consider the evaluation map $E(1): E \rightarrow \mathbb{C}$ defined by $t \underline{m} \mapsto 1$. Denote the composition map $E(1) \circ \psi$ as $\psi$. In what follows, we prove that for such $\psi$ and $\psi$ there exist an irreducible module $V(\Psi)$ and a non-graded irreducible module $V(\psi)$, and we prove that $V \cong V(\psi)$. We will also indicate how to get $V(\psi)$ 
from $V(\Psi)$, and conversely. Thus our problem reduces to classifying $V(\psi)$, and it suffices to describe $\psi$, which is a map from $H \rightarrow \mathbb{C}$.

Nongraded and graded modules for $\tau(\mu)$. We recall from Section 1 that $\Delta=$ $\Delta_{\text {re }} \cup \Delta_{\text {im }}$, and $\Delta=\Delta^{+} \cup \Delta^{0} \cup \Delta^{-}$. Let $\tau_{0}(\mu)=J=(\mathfrak{g} \otimes A)_{\mu} \oplus\left(\Omega_{A} / d_{A}\right)^{0}$. Then $J=J^{+} \oplus J^{0} \oplus J^{-}$, where

$$
J^{+}=\bigoplus_{\alpha \in \Delta^{+}} J_{\alpha}, \quad J^{-}=\bigoplus_{\alpha \in \Delta^{-}} J_{\alpha}, \quad J^{0}=\bigoplus_{\alpha \in \Delta^{0}} J_{\alpha} .
$$

Note that $H=J^{0}$. For any Lie algebra $\mathfrak{g}$, let $U(\mathfrak{g})$ be the universal enveloping algebra.

Let $\psi: J^{0} \rightarrow \mathbb{C}$ be a linear map. We make the one-dimensional vector space $\mathbb{C} v$ a $J^{+} \oplus J^{0} \oplus \mathbb{C} d_{n}$-module by defining

$h . v=\psi(h) v \quad$ for $h \in J^{0}, \quad X v=0 \quad$ for $X \in J^{+}, \quad d_{n} v=d v \quad$ for some $d \in \mathbb{C}$.

We consider the induced Verma module

$$
M(\psi)=U\left(J^{-}\right) \bigotimes_{J^{+} \oplus J^{0} \oplus \mathbb{C} d_{n}} \mathbb{C} v .
$$

From standard arguments, $M(\psi)$ has a proper maximal submodule and hence a unique irreducible quotient $V(\psi)$, which is the non-graded module for $\tau(\mu)$.

We define

$$
\Psi: J^{0} \rightarrow \mathbb{C}\left[t_{1}^{ \pm 1}, \ldots, t_{n-1}^{ \pm 1}\right]=A_{n-1} .
$$

Let $\underline{m}=\left(m_{1}, \ldots, m_{n}\right) \in \mathbb{Z}^{n}$, and let $\underline{m}^{\prime}=\left(m_{1}, \ldots, m_{n-1}\right) \in \mathbb{Z}^{n-1}$. Define

$$
\bar{\psi}\left(h \otimes t^{\underline{m}}\right)=\psi\left(h \otimes t^{\underline{m}}\right) t^{\underline{m}^{\prime}} .
$$

Let $E=$ image $\psi$. Let $\underline{b}=\left(b_{1}, b_{2}, \ldots, b_{n-1}\right) \in \mathbb{C}^{n-1}$. Define a $\tau(\mu)$-module structure on $V(\psi) \otimes A_{n-1}$ by the action

$$
\begin{array}{rlrl}
X \otimes t^{\underline{m}}\left(v \otimes t^{\underline{s}}\right) & =\left(X \otimes t^{\underline{m}} v\right) \otimes t^{\underline{s}+\underline{m}^{\prime}} & \text { for every } v \in V(\psi), \\
& X \otimes t^{\underline{m}} \in \tau_{0}(\mu), t^{\underline{s}} \in A_{n-1}, \\
d_{i} .\left(v \otimes t^{\underline{s}}\right) & =\left(b_{i}+s_{i}\right) v \otimes t^{\underline{s}} & \text { for } 1 \leq i \leq(n-1), \\
d_{n} .\left(v \otimes t^{\underline{s}}\right) & =\left(d_{n} v\right) \otimes t^{\underline{s}} . &
\end{array}
$$

Lemma 3.1. $E$ is an irreducible $J^{0} \oplus D$-module if and only if each nonzero homogeneous element of $E$ is invertible.

Proof. This follows from methods from [Rao 2004].

Proposition 3.2. Let E, $\psi$ and $\psi$ be as above. Suppose every nonzero homogeneous element of $E$ is invertible. Then $V(\psi) \otimes A_{n-1}$ is completely reducible as a $\tau(\mu)$-module, and all components are isomorphic up to a grade shift.

Proof. This follows from methods from [Rao 1995]. 
We recall that $\tau(\mu)=J \oplus D$. Let $\psi, \psi$ and $E$ be as above. Make $E$ a $J^{+} \oplus$ $J^{0} \oplus D$-module by defining that $J^{+}$acts trivially on $E$ and

$$
\begin{aligned}
h \otimes t^{\underline{m}}\left(t^{\underline{s}}\right) & =\psi\left(h \otimes t^{\underline{m}}\right) t^{\underline{\underline{s}}+\underline{m}^{\prime}} & & \text { for } t^{\underline{s}} \in E, h \otimes t^{\underline{m}} \in J^{0}, \\
d_{i} .\left(t^{\underline{\underline{s}}}\right) & =\left(b_{i}+s_{i}\right) t^{\underline{\underline{s}}} & & \text { for } 1 \leq i \leq n-1, \\
d_{n} .\left(t^{\underline{\underline{s}}}\right) & =0 . & &
\end{aligned}
$$

Let $M(\bar{\psi})=U\left(J^{-}\right) \otimes_{J^{+} \oplus J^{0} \oplus D} E$. By standard arguments, let $V(\Psi)$ be the irreducible quotient of $M(\psi) . V(\psi)$ is the graded module for $\tau(\mu)$.

Proposition 3.3. Suppose $E$ is a $J^{0} \oplus D$-irreducible module. Then a component of $V(\psi) \otimes A_{n-1}$ is isomorphic to $V(\Psi)$ as a $\tau(\mu)$-module for some suitable $\underline{b}$.

Proof. The proof is same as in [Rao 2004, Proposition 3.5].

The following is the main result of this paper.

Theorem 3.4. Any irreducible, integrable module for $\tau(\mu)$ with finite-dimensional weight spaces, on which the center acts nontrivially, is an irreducible component of $V(\psi) \otimes A_{n-1}$, where $V(\psi)$ is the non-graded module for $\tau(\mu)$.

Outline of the proof. By Proposition 3.3, there is a one-to-one correspondence between graded and non-graded modules for $\tau(\mu)$. So it suffices to classify the non-graded modules for $\tau(\mu)$. We will prove this theorem in Section 5.

\section{Going to the affine Kac-Moody Lie algebra}

Let $V$ be an irreducible integrable module for $\tau(\mu)$ with finite-dimensional weight spaces. Also we know that if $t^{\underline{m}} K_{i} \neq 0$ on $V$, then $m_{n}=0$ and $i=n$. We have

$$
\begin{aligned}
\tau_{0}(\mu)=\bigoplus_{m \in \mathbb{Z}} \mathfrak{g}_{0} \otimes t_{n}^{2 m} B_{\mu} \bigoplus_{m \in \mathbb{Z}} \mathfrak{g}_{0} \otimes t_{n}^{2 m+1} t_{1} B_{\mu} \\
\bigoplus_{m \in \mathbb{Z}} \mathfrak{g}_{1} \otimes t_{n}^{2 m} t_{1} B_{\mu} \bigoplus_{m \in \mathbb{Z}} \mathfrak{g}_{1} \otimes t_{n}^{2 m+1} B_{\mu} \bigoplus\left(\Omega_{A} / d_{A}\right)^{0} .
\end{aligned}
$$

Let $\mathfrak{g}_{\text {aff }}=\mathfrak{g} \otimes \mathbb{C}\left[t_{n}, t_{n}^{-1}\right] \oplus \mathbb{C} K_{n}$. We define a map

$$
\theta: \tau \rightarrow \mathfrak{g}_{\mathrm{aff}} \otimes \mathbb{C}\left[t_{1}^{ \pm 1}, t_{2}^{ \pm 1}, \ldots, t_{n-1}^{ \pm 1}\right] \oplus D
$$

by

$$
\begin{aligned}
X \otimes t^{\underline{m}} \mapsto\left(X t_{n}^{m_{n}}\right) \otimes t^{\underline{m}^{\prime}}, \quad \text { where } \underline{m}^{\prime}=\left(m_{1}, \ldots, m_{n-1}\right), & \\
t^{\underline{m}} K_{i} & \mapsto \begin{cases}0 & \text { if } i \neq n \text { and } m_{n}=0, \\
K_{n} \otimes t^{\underline{m}} & \text { if } i=n \text { and } m_{n}=0, \\
0 & \text { if } m_{n} \neq 0,\end{cases} \\
d_{i} & \mapsto d_{i} \quad \text { for } 1 \leq i \leq n .
\end{aligned}
$$


It is easy to check that $\theta$ defines a Lie algebra homomorphism and that $\operatorname{ker} \theta \cap \tau(\mu)$ is zero on $V$. Below we give a surjective map $\phi$ from $\mathfrak{g}_{\text {aff }} \otimes \mathbb{C}\left[t_{1}^{ \pm 1}, t_{2}^{ \pm 1}, \ldots, t_{n-1}^{ \pm 1}\right]$ to $\bigoplus_{N} \mathfrak{g}_{\text {aff }}$ (the direct sum of $N$ copies of $\mathfrak{g}_{\text {aff }}$ ) and prove the conditions under which $\phi(\mu)=\phi \mid \tau_{0}(\mu)$ is a surjective map from $\tau_{0}(\mu)$ to $\bigoplus_{N} \mathfrak{g}_{\text {aff }}$. We also prove in Proposition 5.3 that the non-graded module $V(\psi)$ is zero on the kernel of $\phi(\mu)$. Thus $V(\psi)$ is a module for $\bigoplus_{N} \mathfrak{g}_{\text {aff }}$, which is an affine Kac-Moody Lie algebra on which the integrable irreducible modules are tensor products of irreducible highest weight modules [Rao 2005].

Let $n \geq 2$ be a positive integer. For each $i$ in $1 \leq i \leq(n-1)$, let $N_{i}$ be a positive integer. Let $\underline{a}_{i}=\left(a_{i 1}, a_{i 2}, \ldots, a_{i N_{i}}\right)$ be distinct nonzero complex numbers. Let $I=\left(i_{1}, i_{2}, \ldots, i_{n-1}\right)$, where $1 \leq i_{j} \leq N_{j}$. Let $\underline{m}^{\prime}=\left(m_{1}, m_{2}, \ldots, m_{n-1}\right) \in \mathbb{Z}^{n-1}$. Let $\phi$ be a Lie algebra homomorphism defined by

$$
\begin{aligned}
\phi: \mathfrak{g}_{\mathrm{aff}} \otimes \mathbb{C}\left[t_{1}^{ \pm 1}, \ldots, t_{n-1}^{ \pm 1}\right] & \rightarrow\left(\bigoplus_{N=N_{1} N_{2} \cdots N_{n-1} \text {-copies }} \mathfrak{g}_{\mathrm{aff}}\right)=\mathfrak{g}_{\mathrm{aff} N}, \\
X t_{1}^{m_{1}} \cdots t_{n-1}^{m_{n-1}} & \mapsto\left(a_{1 i_{1}}^{m_{1}} a_{2 i_{2}}^{m_{2}} \cdots a_{(n-1) i_{n-1}}^{m_{n-1}} X\right)_{1 \leq i_{1} \leq N_{1} ; \ldots ; 1 \leq i_{n-1} \leq N_{n-1}},
\end{aligned}
$$

where $X \in \mathfrak{g}_{\text {aff }}$.

Following the same proof as in [Rao 2004, Lemma 3.11(a)], we see that $\phi$ is surjective. Let $T \subset\left\{11,12, \ldots, 1 N_{1}\right\}$ be a maximal set such that $a_{1 i}^{2} \neq a_{1 j}^{2}$ whenever $1 i, 1 j \in T$ and $1 \leq i, j \leq N_{1}$.

Proposition 4.1. Let $\phi(\mu)=\phi \mid \tau_{0}(\mu)$. If the cardinality of $T$ is $N_{1}$, then $\phi(\mu)$ is a surjective Lie algebra homomorphism from $\tau_{0}(\mu)$ to $\mathfrak{g}_{\mathrm{aff}}{ }_{N}$.

Proof. We already know that if $m=2$, then $\mathfrak{g}=\mathfrak{g}_{0} \oplus \mathfrak{g}_{1}$. Now there are five cases.

Case 4.1a. Fix $m_{n} \in \mathbb{Z}$, and let $Y_{1}, Y_{2}, \ldots, Y_{N} \in \mathfrak{g}_{0}$. Let

$$
\phi(\mu)\left(\sum_{i=1}^{N} b_{i} X_{i} t_{n}^{2 m_{n}} t_{1}^{2 m_{1}} t_{2}^{m_{2}} \cdots t_{n-1}^{m_{n-1}}\right)=\left(Y_{1} t_{n}^{2 m_{n}}, Y_{2} t_{n}^{2 m_{n}}, \ldots, Y_{N} t_{n}^{2 m_{n}}\right),
$$

where the $b_{i}$ are complex numbers, $X_{i} t_{n}^{2 m_{n}} t_{1}^{2 m_{1}} t_{2}^{m_{2}} \cdots t_{n-1}^{m_{n-1}} \in \tau_{0}(\mu)$ and $0 \leq m_{j} \leq$ $N_{j}-1$ for $j=1, \ldots, n-1$. So, we have to solve the equation

$$
\left(\sum_{i=1}^{N} b_{i} X_{i} t_{n}^{2 m_{n}} a_{1 i_{1}}^{2 m_{1}} a_{2 i_{2}}^{m_{2}} \cdots a_{(n-1) i_{n-1}}^{m_{n-1}}\right)=\left(Y_{1} t_{n}^{2 m_{n}}, Y_{2} t_{n}^{2 m_{n}}, \ldots, Y_{N} t_{n}^{2 m_{n}}\right) .
$$

Writing this in matrix form, we must prove that the matrix

$$
M=\left(a_{1 i_{1}}^{2 m_{1}} a_{2 i_{2}}^{m_{2}} \cdots a_{(n-1) i_{n-1}}^{m_{n-1}}\right)
$$

is invertible, where $\left(i_{1}, i_{2}, \ldots, i_{n-1}\right)$ for $1 \leq i_{j} \leq N_{j}$ determines the rows and $\underline{m}^{\prime}=\left(m_{1}, m_{2}, \ldots, m_{n-1}\right)$, where $0 \leq m_{j} \leq N_{j}-1$ for all $1 \leq j \leq n-1$, determines 
the columns. Observe that $M$ can be written as a tensor product of other matrices as

$$
M=\left(a_{1 i_{1}}^{2 m_{1}}\right)_{1 \leq i_{1} \leq N_{1}, 0 \leq m_{1} \leq N_{1}-1} \otimes M_{2} \otimes \cdots \otimes M_{n-1},
$$

where

$$
M_{j}:=\left(a_{j i_{j}}^{m_{j}}\right)_{1 \leq i_{j} \leq N_{j}, 0 \leq m_{j} \leq N_{j}-1} \text { for } j=2, \ldots, n-1 .
$$

Let $M_{1}=\left(a_{1 i_{1}}^{2 m_{1}}\right)_{1 \leq i_{1} \leq N_{1}, 0 \leq m_{1} \leq N_{1}-1}$. In other upcoming cases, $M_{1}$ will be defined differently, but the other $M_{j}$ will be the same. If the cardinality of $T$ is $N_{1}$, which means the $a_{1 i_{1}}^{2}$ are all distinct for all $1 \leq i_{1} \leq N_{1}$, then $M_{1}$ is a Vandermonde matrix, so it is invertible. Similarly $M_{2}$ is a Vandermonde matrix because $a_{2 i} \neq a_{2 j}$ for all $1 \leq i, j \leq N_{2}$. So $M_{2}$ is invertible. The other $M_{j}$ for $3 \leq j \leq(n-1)$ are likewise invertible. According to a standard result in linear algebra, the determinant of a tensor product of invertible matrices is nonzero. So $M$ is invertible.

Case 4.1b. Fix $m_{n} \in \mathbb{Z}$, and let $Y_{1}, Y_{2}, \ldots, Y_{N} \in \mathfrak{g}_{0}$. Let

$$
\phi(\mu)\left(\sum_{i=1}^{N} b_{i} X_{i} t_{n}^{2 m_{n}+1} t_{1}^{2 m_{1}+1} t_{2}^{m_{2}} \cdots t_{n-1}^{m_{n-1}}\right)=\left(Y_{1} t_{n}^{2 m_{n}+1}, Y_{2} t_{n}^{2 m_{n}+1}, \ldots, Y_{N} t_{n}^{2 m_{n}+1}\right),
$$

where the $b_{i}$ are complex numbers and $X_{i} t_{n}^{2 m_{n}+1} t_{1}^{2 m_{1}+1} t_{2}^{m_{2}} \cdots t_{n-1}^{m_{n-1}} \in \tau_{0}(\mu)$ and $0 \leq m_{j} \leq N_{j}-1$ for $1 \leq j \leq(n-1)$. So we have to solve the equation

$$
\left(\sum_{i=1}^{N} b_{i} X_{i} t_{n}^{2 m_{n}+1} a_{1 i_{1}}^{2 m_{1}+1} a_{2 i_{2}}^{m_{2}} \cdots a_{(n-1) i_{n-1}}^{m_{n-1}}\right)=\left(Y_{1} t_{n}^{2 m_{n}+1}, Y_{2} t_{n}^{2 m_{n}+1}, \ldots, Y_{N} t_{n}^{2 m_{n}+1}\right) .
$$

Writing this in the matrix form, we have to prove that the matrix

$$
M=\left(a_{1 i_{1}}^{2 m_{1}+1} a_{2 i_{2}}^{m_{2}} \cdots a_{(n-1) i_{n-1}}^{m_{n-1}}\right)
$$

is invertible, where $\left(i_{1}, i_{2}, \ldots, i_{n-1}\right), 1 \leq i_{j} \leq N_{j}$ determines rows and $\underline{m}^{\prime}=$ $\left(m_{1}, m_{2}, \ldots, m_{n-1}\right)$, where $0 \leq m_{j} \leq N_{j}-1$ for all $1 \leq j \leq(n-1)$, determines the columns. The matrix $M$ is a tensor product of other matrices:

$$
M=\left(a_{1 i_{1}}^{2 m_{1}+1}\right)_{1 \leq i_{1} \leq N_{1}, 0 \leq m_{1} \leq N_{1}-1} \otimes M_{2} \otimes \cdots \otimes M_{n-1} .
$$

Let $M_{1}=\left(a_{1 i_{1}}^{2 m_{1}+1}\right)_{1 \leq i_{1} \leq N_{1}, 0 \leq m_{1} \leq N_{1}-1}$. Again as above, if the cardinality of $T$ is $N_{1}$, then $M_{1}$ is product of two matrices: one is diagonal matrix whose diagonal entries are $a_{11}, a_{12}, \ldots, a_{1 N_{1}}$ and other is $\left(a_{1 i_{1}}^{2 m_{1}}\right)_{1 \leq i_{1} \leq N_{1}, 0 \leq m_{1} \leq N_{1}-1}$. So $M_{1}$ is invertible because the diagonal matrix is invertible and because the other matrix is a Vandermonde matrix. Similarly the $M_{j}$ for $2 \leq j \leq(n-1)$ are invertible since they are Vandermonde matrices. Hence $M$ is invertible. 
Case 4.1c. Fix $m_{n} \in \mathbb{Z}$, and let $Y_{1}, Y_{2}, \ldots, Y_{N} \in \mathfrak{g}_{1}$. Let

$$
\phi(\mu)\left(\sum_{i=1}^{N} b_{i} X_{i} t_{n}^{2 m_{n}} t_{1}^{2 m_{1}+1} t_{2}^{m_{2}} \cdots t_{n-1}^{m_{n-1}}\right)=\left(Y_{1} t_{n}^{2 m_{n}}, Y_{2} t_{n}^{2 m_{n}}, \ldots, Y_{N} t_{n}^{2 m_{n}}\right),
$$

where the $b_{i}$ are complex numbers and $X_{i} t_{n}^{2 m_{n}} t_{1}^{2 m_{1}+1} t_{2}^{m_{2}} \cdots t_{n-1}^{m_{n-1}} \in \tau_{0}(\mu)$ and $0 \leq$ $m_{j} \leq N_{j}-1$ for $\left.1 \leq j \leq(n-1)\right)$. As above we must solve the equation

$$
\left(\sum_{i=1}^{N} b_{i} X_{i} t_{n}^{2 m_{n}} a_{1 i_{1}}^{2 m_{1}+1} a_{2 i_{2}}^{m_{2}} \cdots a_{(n-1) i_{n-1}}^{m_{n-1}}\right)=\left(Y_{1} t_{n}^{2 m_{n}}, Y_{2} t_{n}^{2 m_{n}}, \ldots, Y_{N} t_{n}^{2 m_{n}}\right) .
$$

For this we must prove that the matrix

$$
M=\left(a_{1 i_{1}}^{2 m_{1}+1} a_{2 i_{2}}^{m_{2}} \cdots a_{(n-1) i_{n-1}}^{m_{n-1}}\right),
$$

is invertible, where $\left(i_{1}, i_{2}, \ldots, i_{n-1}\right)$ for $1 \leq i_{j} \leq N_{j}$ determines the rows and $\underline{m}^{\prime}=\left(m_{1}, m_{2}, \ldots, m_{n-1}\right)$, where $0 \leq m_{j} \leq N_{j}-1$ for all $1 \leq j \leq(n-1)$, determines the columns. Again by Case 4.1b, $M$ is invertible if the cardinality of $T$ is $N_{1}$.

Case 4.1d. Fix $m_{n} \in \mathbb{Z}$, and let $Y_{1}, Y_{2}, \ldots, Y_{N} \in \mathfrak{g}_{1}$. Let

$$
\phi(\mu)\left(\sum_{i=1}^{N} b_{i} X_{i} t_{n}^{2 m_{n}+1} t_{1}^{2 m_{1}} t_{2}^{m_{2}} \cdots t_{n-1}^{m_{n-1}}\right)=\left(Y_{1} t_{n}^{2 m_{n}+1}, Y_{2} t_{n}^{2 m_{n}+1}, \ldots, Y_{N} t_{n}^{2 m_{n}+1}\right),
$$

where the $b_{i}$ are complex numbers and $X_{i} t_{n}^{2 m_{n}+1} t_{1}^{2 m_{1}} t_{2}^{m_{2}} \cdots t_{n-1}^{m_{n-1}} \in \tau_{0}(\mu)$ and $0 \leq$ $m_{j} \leq N_{j}-1$ for $1 \leq j \leq(n-1)$. So we have to solve the equation

$$
\left(\sum_{i=1}^{N} b_{i} X_{i} t_{n}^{2 m_{n}+1} a_{1 i_{1}}^{2 m_{1}} a_{2 i_{2}}^{m_{2}} \cdots a_{(n-1) i_{n-1}}^{m_{n-1}}\right)=\left(Y_{1} t_{n}^{2 m_{n}+1}, Y_{2} t_{n}^{2 m_{n}+1}, \ldots, Y_{N} t_{n}^{2 m_{n}+1}\right) .
$$

For this we must prove the matrix

$$
M=\left(a_{1 i_{1}}^{2 m_{1}} a_{2 i_{2}}^{m_{2}} \cdots a_{(n-1) i_{n-1}}^{m_{n-1}}\right)
$$

is invertible, where $\left(i_{1}, i_{2}, \ldots, i_{n-1}\right)$ for $1 \leq i_{j} \leq N_{j}$ determines the rows and $\underline{m}^{\prime}=\left(m_{1}, m_{2}, \ldots, m_{n-1}\right)$, where $0 \leq m_{j} \leq N_{j}-1$ for all $1 \leq j \leq(n-1)$, determines the columns. Again, by Case 4.1a, $M$ is invertible if the cardinality of $T$ is $N_{1}$.

Case 4.1e. Let $c_{1}, c_{2}, \ldots, c_{N} \in \mathbb{C}$. Let

$$
\phi(\mu)\left(\sum_{i=1}^{N} b_{i} K_{n} t_{1}^{2 m_{1}} t_{2}^{m_{2}} \cdots t_{n-1}^{m_{n-1}}\right)=\left(c_{1} K_{n}, c_{2} K_{n}, \ldots, c_{N} K_{n}\right),
$$


where the $b_{i}$ are complex numbers and $K_{n} t_{1}^{2 m_{1}} t_{2}^{m_{2}} \cdots t_{n-1}^{m_{n-1}} \in \tau_{0}(\mu)$ and $0 \leq m_{j} \leq$ $N_{j}-1$ for $1 \leq j \leq(n-1)$. So we must solve

$$
\left(\sum_{i=1}^{N} b_{i} K_{n} a_{1 i_{1}}^{2 m_{1}} a_{2 i_{2}}^{m_{2}} \cdots a_{(n-1) i_{n-1}}^{m_{n-1}}\right)=\left(c_{1} K_{n}, c_{2} K_{n}, \ldots, c_{N} K_{n}\right) .
$$

So, we have to prove that the matrix

$$
M=\left(a_{1 i_{1}}^{2 m_{1}} a_{2 i_{2}}^{m_{2}} \cdots a_{(n-1) i_{n-1}}^{m_{n-1}}\right)
$$

is invertible, where $\left(i_{1}, i_{2}, \ldots, i_{n-1}\right)$ for $1 \leq i_{j} \leq N_{j}$ determines the rows and $\underline{m}^{\prime}=\left(m_{1}, m_{2}, \ldots, m_{n-1}\right)$, where $0 \leq m_{j} \leq N_{j}-1$ for all $1 \leq j \leq(n-1)$, determines the columns. Again by Case 4.1a, $M$ is invertible if the cardinality of $T$ is $N_{1}$.

So if the cardinality of $T$ is $N_{1}$, then $\phi(\mu)$ is a surjective Lie algebra homomorphism from $\tau_{0}(\mu)$ to $\mathfrak{g}_{\text {aff }}$. This completes the proof of Proposition 4.1.

We now define polynomials $P_{1}\left(t_{1}^{2}\right), P_{2}\left(t_{2}\right), \ldots, P_{n-1}\left(t_{n-1}\right)$ by

$$
P_{1}\left(t_{1}^{2}\right)=\prod_{i=1}^{N_{1}}\left(t_{1}^{2}-a_{1 i}^{2}\right), \quad P_{j}\left(t_{j}\right)=\prod_{l=1}^{N_{j}}\left(t_{j}-a_{j l}\right) \quad \text { for } 2 \leq j \leq(n-1) .
$$

Notation. Denote by $\left\langle P_{1}\left(t_{1}^{2}\right), P_{2}\left(t_{2}\right), \ldots, P_{n-1}\left(t_{n-1}\right)\right\rangle$ the ideal generated by the polynomials $P_{1}\left(t_{1}^{2}\right), P_{2}\left(t_{2}\right), \ldots, P_{n-1}\left(t_{n-1}\right)$ inside $\mathbb{C}\left[t_{1}^{ \pm 2}, t_{2}^{ \pm 1}, \ldots, t_{n-1}^{ \pm 1}\right]$.

Lemma 4.2. If the cardinality of $T$ is $N_{1}$, then $\operatorname{ker} \phi(\mu)$ is given by $I_{1}+I_{2}+I_{3}+$ $I_{4}+I_{5}$, where, for some fixed $m \in \mathbb{Z}$,

$$
\begin{aligned}
& I_{1}=\mathfrak{g}_{0} \otimes t_{n}^{2 m} \quad\left\langle P_{1}\left(t_{1}^{2}\right), P_{2}\left(t_{2}\right), \ldots, P_{n-1}\left(t_{n-1}\right)\right\rangle, \\
& I_{2}=\mathfrak{g}_{0} \otimes t_{n}^{2 m+1}\left\langle t_{1} P_{1}\left(t_{1}^{2}\right), P_{2}\left(t_{2}\right), \ldots, P_{n-1}\left(t_{n-1}\right)\right\rangle, \\
& I_{3}=\mathfrak{g}_{1} \otimes t_{n}^{2 m} \quad\left\langle t_{1} P_{1}\left(t_{1}^{2}\right), P_{2}\left(t_{2}\right), \ldots, P_{n-1}\left(t_{n-1}\right)\right\rangle, \\
& I_{4}=\mathfrak{g}_{1} \otimes t_{n}^{2 m+1}\left\langle P_{1}\left(t_{1}^{2}\right), P_{2}\left(t_{2}\right), \ldots, P_{n-1}\left(t_{n-1}\right)\right\rangle, \\
& I_{5}=K_{n} \quad\left\langle P_{1}\left(t_{1}^{2}\right), P_{2}\left(t_{2}\right), \ldots, P_{n-1}\left(t_{n-1}\right)\right\rangle .
\end{aligned}
$$

Proof. We consider the map

$$
\begin{aligned}
\phi_{1}: \mathfrak{g}_{0} \otimes t_{n}^{2 m} t^{\underline{m}^{\prime}} & \rightarrow \bigoplus_{N=N_{1} N_{2} \cdots \cdot N_{n-1}} \mathfrak{g}_{0} \otimes t_{n}^{2 m}, \quad \text { where } t^{\underline{m}^{\prime}} \in B_{\mu}, \\
X t_{n}^{2 m} t^{\underline{m}^{\prime}} & \mapsto\left(X t_{n}^{2 m} a_{1 i_{1}}^{2 m_{1}} a_{2 i_{2}}^{m_{2}} \cdots a_{(n-1) i_{n-1}}^{m_{n-1}}\right)_{1 \leq i_{1} \leq N_{1} ; \ldots ; 1 \leq i_{n-1} \leq N_{n-1}} .
\end{aligned}
$$

Let $M=\left(N=N_{1} N_{2} \cdots N_{n-1}\right)$ (the dimension of $\left.\mathfrak{g}_{0}\right)$. As proved in Proposition 4.1, one can show that $\phi_{1}$ is surjective. Since $\phi_{1}$ is nothing but the evaluation 
map at the roots of $P_{1}\left(t_{1}^{2}\right), P_{2}\left(t_{2}\right), \ldots, P_{n-1}\left(t_{n-1}\right)$, we have $I_{1} \subset \operatorname{ker} \phi_{1}$. Therefore $\operatorname{dim}\left(\mathfrak{g}_{0} \otimes t_{n}^{2 m} t^{\underline{m^{\prime}}} / I_{1}\right) \geq M$. Consider the space

$$
R=\left\{\mathfrak{g}_{0} \otimes t_{n}^{2 m} t_{1}^{2 m_{1}} t_{2}^{m_{2}} \cdots t_{n-1}^{m_{n-1}} \mid 0 \leq m_{i} \leq N_{i}-1 \quad \text { for } 1 \leq i \leq(n-1)\right\} .
$$

Note that $\phi_{1}$ is injective on $R$ since the corresponding matrix is invertible. Also note that any element of $\mathfrak{g}_{0} \otimes t_{n}^{2 m} t^{\underline{m}^{\prime}}$ can be written as linear combination of elements of $R$ modulo $I_{1}$. Thus $\operatorname{dim}\left(\mathfrak{g}_{0} \otimes t_{n}^{2 m} t^{\underline{m^{\prime}}} / I_{1}\right) \leq M$, and $\operatorname{sodim}\left(\mathfrak{g}_{0} \otimes t_{n}^{2 m} t^{\underline{m^{\prime}}} / I_{1}\right)=M$. Therefore $I_{1}$ is the kernel of $\phi_{1}$. Arguing similarly on the other components of $\tau(\mu)$, we get that $I_{1}+I_{2}+I_{3}+I_{4}+I_{5}$ is the kernel of $\phi(\mu)$ if the cardinality of $T$ is $N_{1}$.

\section{Proof of the main theorem and propositions}

Proof of Theorem 3.4. Let $V$ be an irreducible integrable module for $\tau(\mu)$ with finite-dimensional weight spaces. Then, by Theorems 2.3 and 2.4, there exist up to a coordinate change nonzero central operators $Z_{1}, \ldots, Z_{n-1}$ of respective degrees $\left(k_{1}, 0, \ldots, 0\right), \ldots,\left(0, \ldots, k_{n-1}, 0\right)$. Let $W=\left\{Z_{i} v-v \mid v \in V\right.$ for $\left.1 \leq i \leq(n-1)\right\}$. We claim that $W$ is a maximal proper $J \oplus \mathbb{C} d_{n}$-submodule of $V$.

Consider $W_{1}=\left\{Z_{1} v-v \mid v \in V\right\}$. $W_{1}$ is a proper $J \oplus D_{1}$-module. Note that if $i \neq 1$, then $d_{i}$ commutes with $Z_{1}$, and hence $W_{1}$ is $d_{i}$-invariant. Clearly $W_{1}$ is $J$-invariant. To see that $W_{1}$ is proper, just note that $W_{1}$ cannot contain $d_{1}$ weight vectors. Now consider $W_{2}=\left\{Z_{2} v-v \mid v \in V / W_{1}\right\}$. By the above argument, we can see that $W_{2}$ is a proper $J \oplus D_{2}$-module. Continuing this process, we see that $W=\left\{Z_{i} v-v \mid v \in V\right.$ for $\left.1 \leq i \leq(n-1)\right\}$ is a maximal proper $J \oplus \mathbb{C} d_{n}$-submodule of $V$.

Thus we have proved that $W$ is a maximal proper $J \oplus \mathbb{C} d_{n}$-submodule of $V$ and $V / W$ has finite-dimensional weight spaces with respect to $\mathfrak{h}(\mu) \oplus \sum_{i=1}^{n} \mathbb{C} K_{i} \oplus$ $\mathbb{C} d_{n}$. Further, we proved in Proposition 2.6 that there exists a $\lambda \in P(V)$ such that $\lambda+\eta+\delta_{\underline{m}^{\prime}} \notin P(V)$ for every $\eta>0$ and for every $\underline{m}^{\prime} \in \mathbb{Z}^{n-1}$.

Let $\bigoplus_{\underline{m}^{\prime}} V_{\lambda+\delta_{\underline{m}^{\prime}}}=V^{\lambda}$. Then it follows that

$$
X_{\alpha} \otimes t^{\underline{s}} V^{\lambda}=0 \quad \text { for all } \alpha+\delta_{\underline{s}} \in \Delta_{\mathrm{re}}^{+} .
$$

Let $\bar{V}^{\lambda}$ be the image of $V^{\lambda}$ in $V / W$, which is known to be finite-dimensional. $J^{0}$ leaves $\bar{V}^{\lambda}$ invariant. Notice that $J^{0}$ is an abelian Lie algebra. Thus $J^{0}$ has a one-dimensional invariant subspace in $\bar{V}^{\lambda}$. Let $W^{\lambda}$ be the maximal proper invariant subspace of $\bar{V}^{\lambda}$. Then it is easy to check that $\bar{V}^{\lambda} / W^{\lambda}$ is one-dimensional by Lie's theorem; otherwise there will be a contradiction to the fact that $W^{\lambda}$ is a maximal proper subspace. Let $\bar{V}^{\lambda} / W^{\lambda}=\mathbb{C} v$. Let $X \otimes t^{\underline{m}} v=\psi\left(X \otimes t^{\underline{m}}\right) v$ for $X \otimes t^{\underline{m}} \in J^{0}$, where $\psi: J^{0} \rightarrow \mathbb{C}$ is a linear map. Now consider the irreducible module $V(\psi)$ as described in Section 3. 
We claim that $V(\psi)$ is a $\tau_{0}(\mu) \oplus \mathbb{C} d_{n}$-irreducible quotient of $V / W$. This follows from the fact that the module generated by $W^{\lambda}$ is proper and maximal. Thus we have an irreducible non-graded module $V(\psi)$ for $\tau_{0}(\mu) \oplus \mathbb{C} d_{n}$. Now $V(\psi) \otimes A_{n-1}$ can be made as a $\tau(\mu)$-module as described in Section 3. Now by Propositions 3.2 and 3.3, it follows that any irreducible integrable module for $\tau(\mu)$ with finitedimensional weight spaces is an irreducible component of $V(\psi) \otimes A_{n-1}$. This completes the proof of Theorem 3.4.

In case $m=2$, we have $B_{\mu}=\mathbb{C}\left[t_{1}^{ \pm 2}, t_{2}^{ \pm 1}, \ldots, t_{n-1}^{ \pm 1}\right]$ and $\Gamma=2 \underline{e_{1}} \mathbb{Z} \oplus \underline{e_{2}} \mathbb{Z} \oplus \cdots \oplus$ $e_{n-1} \mathbb{Z}$. Notice that $t^{\underline{m}^{\prime}} \in B_{\mu}$ if and only if $\underline{m}^{\prime} \in \Gamma$. Let

$$
\begin{aligned}
T_{1} & =\bigoplus_{m \in \mathbb{Z}} \mathfrak{g}_{0} \otimes t_{n}^{2 m} B_{\mu}, & T_{2} & =\bigoplus_{m \in \mathbb{Z}} \mathfrak{g}_{0} \otimes t_{n}^{2 m+1} t_{1} B_{\mu}, \\
T_{3} & =\bigoplus_{m \in \mathbb{Z}} \mathfrak{g}_{1} \otimes t_{n}^{2 m} t_{1} B_{\mu}, & T_{4} & =\bigoplus_{m \in \mathbb{Z}} \mathfrak{g}_{1} \otimes t_{n}^{2 m+1} B_{\mu} .
\end{aligned}
$$

So $\tau(\mu)=T_{1} \oplus T_{2} \oplus T_{3} \oplus T_{4} \oplus\left(\Omega_{A} / d_{A}\right)^{0} \oplus D$. Let $T_{i}^{+}=T_{i} \bigcap\left(\bigoplus_{\alpha \in \Delta^{+}} \tau_{\alpha}\right)$. Let $\alpha_{1}, \ldots, \alpha_{l}$ be all simple roots of $\mathfrak{g}_{0}$, and let $\alpha_{i_{1}}, \ldots, \alpha_{i_{s}}$ be all positive short roots of $\mathfrak{g}_{1}$. Let

$$
\begin{aligned}
S=\left\{\alpha_{1}+\delta_{\underline{m}^{\prime}}, \ldots, \alpha_{l}+\delta_{\underline{m}^{\prime}}, \underline{m}^{\prime} \in \Gamma,\right. & \\
-\alpha_{1}+\delta_{n}+\delta_{1}+\delta_{\underline{m}^{\prime}}, \ldots, & -\alpha_{l}+\delta_{n}+\delta_{1}+\delta_{\underline{m}^{\prime}}, \delta_{n}+\delta_{1}+\delta_{\underline{m}^{\prime}}, \underline{m}^{\prime} \in \Gamma, \\
\alpha_{i_{1}}+\delta_{1}+\delta_{\underline{m}^{\prime}} & \ldots, \alpha_{i_{s}}+\delta_{1}+\delta_{\underline{m}^{\prime}}, \underline{m}^{\prime} \in \Gamma, \\
& \left.-\alpha_{i_{1}}+\delta_{n}+\delta_{\underline{m}^{\prime}}, \ldots,-\alpha_{i_{s}}+\delta_{n}+\delta_{\underline{m}^{\prime}}, \underline{m}^{\prime} \in \Gamma\right\} .
\end{aligned}
$$

Proposition 5.1. Let $\langle S\rangle$ be the Lie algebra generated by the root spaces corresponding to root $\alpha \in S$. Then

$$
\langle S\rangle=T_{1}^{+} \oplus T_{2}^{+} \oplus T_{3}^{+} \oplus T_{4}^{+}=\bigoplus_{\alpha \in \Delta^{+}} \tau_{\alpha} .
$$

Proof. Let

$$
\tau_{1}(\mu)=\bigoplus_{m \in \mathbb{Z}} \mathfrak{g}_{0} \otimes t_{n}^{2 m} \bigoplus_{m \in \mathbb{Z}} \mathfrak{g}_{1} \otimes t_{n}^{2 m+1} \bigoplus \mathbb{C} K_{n},
$$

which is a twisted affine Lie algebra. Let $\beta$ be the weight corresponding to the highest weight vector of the $\mathfrak{g}_{0}$-module $\mathfrak{g}_{1}$ as in [Kac 1990]. Then it is known from [Kac 1990] that $\tau_{1}(\mu)$ is generated as a Lie algebra by the root spaces corresponding to the root basis $\left\{\alpha_{1}, \ldots, \alpha_{l},-\beta+\delta_{n}\right\}$. Thus from this we conclude that $\langle S\rangle$ contains $T_{1}^{+} \oplus T_{4}^{+}$, which is the Lie algebra generated by the root spaces corresponding to the roots $\alpha_{1}+\delta_{\underline{m}^{\prime}}, \ldots, \alpha_{l}+\delta_{\underline{m}^{\prime}},-\beta+\delta_{n}+\delta_{\underline{m}^{\prime}}$ for $\underline{m}^{\prime} \in \Gamma$. Also

$$
\left[\mathfrak{h}(\mu) t_{1} t_{n}, T_{1}^{+}\right]=T_{2}^{+} \quad \text { and } \quad\left[\mathfrak{h}(\mu) t_{1} t_{n}, T_{4}^{+}\right]=t_{n}{ }^{2} T_{3}^{+},
$$


and the root spaces corresponding to the roots $\alpha_{i_{1}}+\delta_{1}+\delta_{\underline{m}^{\prime}}, \ldots, \alpha_{i_{s}}+\delta_{1}+\delta_{\underline{m}^{\prime}}$ for $\underline{m}^{\prime} \in \Gamma$ are in $\langle S\rangle$ by definition. Thus $\langle S\rangle=T_{1}^{+} \oplus T_{2}^{+} \oplus T_{3}^{+} \oplus T_{4}^{+}$.

We will now define the concept of the height ht of a positive root. All roots of $\mathfrak{g}_{0}$ are given the usual height. Thus if $\alpha=\sum n_{i} \alpha_{i}$, then ht $\alpha=\sum n_{i}$. Also ht $\delta_{n}=N+1$, where $N$ is the height of the highest root of the finite root system $\Delta_{0}$ of $\mathfrak{g}_{0}$. Let $\alpha \in \Delta^{+}$. Let $\alpha=\alpha^{\prime}+\delta_{\underline{m}^{\prime}}$ and $m_{n}=0$. Then we define ht $\alpha=$ ht $\alpha^{\prime}$. Let

$$
\begin{aligned}
\bar{S}=\left\{\alpha_{1}, \ldots, \alpha_{l},-\alpha_{1}+\delta_{n}+\delta_{1}, \ldots,-\alpha_{l}+\delta_{n}+\delta_{1}, \delta_{1}+\delta_{n},\right. & \\
& \left.\alpha_{i_{1}}+\delta_{1}, \ldots, \alpha_{i_{s}}+\delta_{1},-\alpha_{i_{1}}+\delta_{n}, \ldots,-\alpha_{i_{s}}+\delta_{n}\right\} .
\end{aligned}
$$

Clearly ht $\gamma \leq N+1$ for every $\gamma \in \bar{S}$. It is easy to see that for $\gamma^{\prime} \in \Delta^{+}$, there exists a $\gamma \in \bar{S}$ such that $\gamma^{\prime}=\gamma+\delta_{\underline{m}^{\prime}}$ for $\underline{m}^{\prime} \in \Gamma$ and ht $\gamma \leq N+1$.

Lemma 5.2. Suppose $V(\psi)$ has finite-dimensional weight spaces. Then $X_{\alpha} \otimes$ $I V(\psi)=0$ for some cofinite ideal I of $\mathbb{C}\left[t_{1}^{ \pm 2}, t_{2}^{ \pm 1} \cdots t_{n-1}^{ \pm 1}\right]$ and for all $\alpha \in \Delta$, including $\alpha=0$.

Proof. Let $X_{\alpha}$ be a root vector of root $\alpha \in-\bar{S}$. For $2 \leq i \leq(n-1)$, consider the set $\left\{X_{\alpha} \otimes t_{i}^{k} v \mid k \in \mathbb{Z}\right\}$, which is contained in the same weight space. Since weight spaces are finite-dimensional, there exists a nonzero polynomial $P_{i, \alpha}\left(t_{i}\right)=\sum_{j} a_{j} t_{i}^{j}$ such that

$$
X_{\alpha} \otimes P_{i, \alpha}\left(t_{i}\right) v=0, \quad \text { where } X_{\alpha} \otimes P_{i, \alpha}\left(t_{i}\right)=\sum_{j} a_{j} X_{\alpha} \otimes t_{i}^{j} .
$$

Denote by $(P)$ the ideal generated by the polynomial $P$ in $\mathbb{C}\left[t_{1}^{ \pm 1}, t_{2}^{ \pm 1}, \ldots, t_{n-1}^{ \pm 1}\right]$.

Claim 5.2a. $X_{\alpha} \otimes\left(P_{i, \alpha}\left(t_{i}\right)\right) v=0$ for $2 \leq i \leq n-1$. Let $\underline{m}^{\prime} \in B_{\mu}$. Then

$$
h \otimes t^{\underline{m}^{\prime}}\left(X_{\alpha} \otimes P_{i, \alpha}\left(t_{i}\right)\right) v=X_{\alpha} \otimes P_{i, \alpha}\left(t_{i}\right) h \otimes t^{\underline{m}^{\prime}} v+\alpha(h) X_{\alpha} \otimes t^{\underline{m}^{\prime}} P_{i, \alpha}\left(t_{i}\right) v=0 .
$$

The first term is zero since $h \otimes t \underline{\underline{m}}^{\prime}$ acts as scalar. Thus

$$
X_{\alpha} \otimes t^{\underline{m}^{\prime}}\left(P_{i, \alpha}\left(t_{i}\right)\right) v=0 \quad \text { for } 2 \leq i \leq n-1 .
$$

Let $P_{i}\left(t_{i}\right)=\prod_{-\alpha \in \bar{S}} P_{i, \alpha}\left(t_{i}\right)$. We note that $X_{\alpha} \otimes B_{\mu}\left(P_{i}\left(t_{i}\right)\right) v=0$ for $2 \leq i \leq n-1$. Similarly for $t_{1}$, consider the set $\left\{X_{\alpha} \otimes t_{1}^{2 k} v \mid k \in \mathbb{Z}\right\}$, which is contained in the same weight space. Since weight spaces are finite-dimensional, there exists a nonzero polynomial $P_{1, \alpha}\left(t_{1}\right)=\sum_{j} a_{2 j} t_{1}^{2 j}$ such that $X_{\alpha} \otimes P_{1, \alpha}\left(t_{1}\right) v=0$. On similar lines as above, we prove that

$$
X_{\alpha} \otimes t^{\underline{m}^{\prime}}\left(P_{1, \alpha}\left(t_{1}\right)\right) v=0 \quad \text { for } \underline{m}^{\prime} \in \Gamma .
$$

So we have proved that $X_{\alpha} \otimes B_{\mu}\left(P_{i}\left(t_{i}\right)\right) v=0$ for $1 \leq i \leq n-1$. 
Claim 5.2b. For $1 \leq i \leq n-1$,

$X_{\gamma} \otimes\left(P_{i}\left(t_{i}\right)\right) v=0 \quad$ for every $\gamma \in-\Delta^{+}, \quad$ where $\gamma=\eta+\delta_{\underline{m}^{\prime}}$ and $m_{n}=0$.

We will prove this by induction on the height of a positive root. We have already seen this by Claim 5.2a for all $\gamma \in-\bar{S}$ such that ht $\gamma \leq$ ht $\delta_{n}$. Let $\gamma \in-\Delta^{+}$, where $\gamma=\eta+\delta_{\underline{m}^{\prime}}$ and $m_{n}=0$, and consider $X_{\gamma} \otimes\left(P_{i}\left(t_{i}\right)\right) v$ for $1 \leq i \leq(n-1)$. We will prove that $X_{\gamma} \otimes\left(P_{i}\left(t_{i}\right)\right) v$ is killed by $X_{\beta}$ for every $\beta \in S$. Then by Proposition 5.1, it follows that $X_{\gamma} \otimes\left(P_{i}\left(t_{i}\right)\right) v$ is killed by $X_{\beta}$ for every $\beta \in \Delta^{+}$, since $S$ generates all the positive root spaces. So $X_{\gamma} \otimes\left(P_{i}\left(t_{i}\right)\right) v$ is a highest weight vector. Since $V(\psi)$ is an irreducible highest weight module, it follows that $X_{\gamma} \otimes\left(P_{i}\left(t_{i}\right)\right) v=0$ for every $\gamma \in-\Delta^{+}$, where $\gamma=\eta+\delta_{\underline{m}^{\prime}}$ and $m_{n}=0$.

Let $\beta \in S$, and let $\beta=\alpha+\delta_{\underline{m}^{\prime}}$ for $\alpha \in \bar{S}$ and $\underline{m}^{\prime} \in \Gamma$. Then

$$
X_{\alpha} \otimes t^{\underline{m}^{\prime}} . X_{\gamma} \otimes\left(P_{i}\left(t_{i}\right)\right) v=X_{\gamma} \otimes\left(P_{i}\left(t_{i}\right)\right) . X_{\alpha} \otimes t^{\underline{m^{\prime}}} v+\left[X_{\alpha}, X_{\gamma}\right] \otimes t^{\underline{m^{\prime}}}\left(P_{i}\left(t_{i}\right)\right) v .
$$

The first component is zero since $v$ is a highest weight vector. The second is zero by induction on the height of a positive root. This proves Claim 5.2b.

Claim 5.2c. $b \otimes\left(P_{i}\left(t_{i}\right)\right) v=0$ for every $b \in J^{0}$ and for $1 \leq i \leq(n-1)$.

For any $\alpha \in-\Delta_{\mathrm{re}}^{+}$, consider $-\alpha^{\vee} \otimes\left(P_{i}\left(t_{i}\right)\right) v=X_{-\alpha} X_{\alpha} \otimes\left(P_{i}\left(t_{i}\right)\right) v-X_{\alpha} \otimes$ $\left(P_{i}\left(t_{i}\right)\right) X_{-\alpha} v=0$ by Claim 5.2b. Since $\alpha^{\vee}$ for $\alpha \in-\Delta_{\text {re }}^{+}$will cover all of $J^{0}$, this proves Claim 5.2c.

Let $I$ be the ideal $P_{1}\left(t_{1}\right), \ldots, P_{n-1}\left(t_{n-1}\right)$ generates inside $\mathbb{C}\left[t_{1}^{ \pm 2}, t_{2}^{ \pm 1} \cdots t_{n-1}^{ \pm 1}\right]$. It is easy to see that $\mathbb{C}\left[t_{1}^{ \pm 2}, t_{2}^{ \pm 1} \cdots t_{n-1}^{ \pm 1}\right] / I$ is finite-dimensional. We consider

$$
W=\left\{w \in V(\psi) \mid X_{\alpha} \otimes I w=0 \text { for all } \alpha \text {, including } \alpha=0\right\},
$$

which is nonempty by the above. It is easy to see that $W$ is a submodule of $V(\psi)$. Since $V(\psi)$ is irreducible, $W=V(\psi)$. This completes the proof of Lemma 5.2.

Let $I^{\prime}$ be the ideal generated by the polynomials $P_{1}\left(t_{1}^{2}\right), \ldots, P_{n-1}\left(t_{n-1}\right)$ inside $\mathbb{C}\left[t_{1}^{ \pm 2}, t_{2}^{ \pm 1}, \ldots, t_{n-1}^{ \pm 1}\right]$. That is, let

$$
I^{\prime}=\left\langle P_{1}\left(t_{1}^{2}\right)=\prod_{i=1}^{N_{1}}\left(t_{1}^{2}-a_{1 i}^{2}\right), \quad P_{j}\left(t_{j}\right)=\prod_{l=1}^{N_{j}}\left(t_{j}-a_{j l}\right) \quad \text { for } 2 \leq j \leq(n-1)\right\rangle .
$$

We observe that the polynomials $P_{1}\left(t_{1}^{2}\right), \ldots, P_{n-1}\left(t_{n-1}\right)$ have distinct roots. So $I \subset I^{\prime}$. Our aim is to prove that $X_{\alpha} \otimes I^{\prime} v=0$ for all $\alpha$, including $\alpha=0$.

Let $\alpha$ be a simple root of $\mathfrak{g}_{0}$.

Case 5.2d. Suppose $\alpha$ is a long root.

Let $X_{\alpha} \in\left(\mathfrak{g}_{0}\right)_{\alpha}$ and $Y_{\alpha} \in\left(\mathfrak{g}_{0}\right)_{-\alpha}$ such that $h_{\alpha}=\left[X_{\alpha}, Y_{\alpha}\right]$ is an $\mathrm{sl}_{2}$-copy. Let $G_{\alpha}$ be the space spanned by $X_{\alpha} \otimes I^{\prime}, Y_{\alpha} \otimes I^{\prime}, h_{\alpha} \otimes I^{\prime}$, and $K_{n} \otimes I^{\prime}$. 
Case 5.2e. Suppose $\alpha$ is a short root.

Let $X_{\alpha} \in\left(\mathfrak{g}_{0}\right)_{\alpha}$ and $Y_{\alpha} \in\left(\mathfrak{g}_{0}\right)_{-\alpha}$ such that $h_{\alpha}=\left[X_{\alpha}, Y_{\alpha}\right]$ is an $\mathrm{sl}_{2}$-copy. Let $X_{\alpha}^{\prime} \in\left(\mathfrak{g}_{1}\right)_{\alpha}$ and $Y_{\alpha}^{\prime} \in\left(\mathfrak{g}_{1}\right)_{-\alpha}$ such that $h_{\alpha}^{\prime}=\left[X_{\alpha}^{\prime}, Y_{\alpha}^{\prime}\right]$ is an $\mathrm{sl}_{2}$-copy. Let $G_{\alpha}$ be the space spanned by

$$
\begin{array}{lrrr}
X_{\alpha} \otimes I^{\prime}, & Y_{\alpha} \otimes I^{\prime}, & h_{\alpha} \otimes I^{\prime}, & X_{\alpha}^{\prime} \otimes t_{1} I^{\prime}, \\
Y_{\alpha}^{\prime} \otimes t_{1}^{-1} I^{\prime}, & h_{\alpha}^{\prime} \otimes I^{\prime}, & {\left[X_{\alpha}, Y_{\alpha}^{\prime} \otimes t_{1}^{-1}\right] \otimes I^{\prime},} & {\left[X_{\alpha}^{\prime} \otimes t_{1}, Y_{\alpha}\right] \otimes I^{\prime} .}
\end{array}
$$

Case 5.2f. Suppose $\beta$ is the highest root of the finite root system $\Delta_{0}$ of $\mathfrak{g}_{0}$.

Consider

$$
\alpha=-\beta+\delta_{n}, \quad X_{\alpha}=X_{-\beta} \otimes t_{n}, \quad Y_{\alpha}=X_{\beta} \otimes t_{n}^{-1}, \quad h_{\alpha}=\left[X_{\alpha}, Y_{\alpha}\right]=h_{\beta}+K_{n} .
$$

Let $G_{\alpha}$ be the space spanned by $X_{\alpha} \otimes I^{\prime}, Y_{\alpha} \otimes I^{\prime}, h_{\alpha} \otimes I^{\prime}$.

Proposition 5.3. Let $\alpha$ be as in Cases 5.2d, 5.2e, and 5.2f. Then $G_{\alpha} v=0$.

Proof. If we take Lie brackets in $G_{\alpha}$, then the result will contain higher powers of $I^{\prime}$. But $I \subset I^{\prime n}$ for large $n$. So $G_{\alpha} / I$ is solvable. In fact it is nilpotent. Since $I^{\prime} / I$ is finite-dimensional, so is $G_{\alpha} / I^{\prime}$. By Lie's theorem, there exists nonzero $w$ in $V(\psi)$ such that $g \otimes p(t) w=\lambda(g, p(t)) w$ for some scalar $\lambda(g, p(t)), g \in G_{\alpha}$, and $p(t) \in I^{\prime}$.

Claim 5.3a. $G_{\alpha} w=0$.

We will prove the proposition for Case 5.2e. For Cases 5.2d and 5.2f, the proof is based on similar arguments. So suppose $\alpha$ is a short root and let $X_{\alpha} \in\left(\mathfrak{g}_{0}\right)_{\alpha}$. Then let $X_{\alpha} \otimes P(t) w=\lambda\left(X_{\alpha}, P(t)\right) w$ for $P(t) \in I^{\prime}$. This implies $\left(X_{\alpha} \otimes P(t)\right)^{m} w=$ $\lambda\left(X_{\alpha}, P(t)\right)^{m} w$. Since $V$ is integrable, $\left(X_{\alpha} \otimes P(t)\right)^{m} w=0$ for large $m$, and therefore $\lambda\left(X_{\alpha}, P(t)\right)=0$. Hence $X_{\alpha} \otimes P(t) w=0$. Similarly we can prove that $Y_{\alpha} \otimes P(t) w=0$. We claim that $h_{\alpha} \otimes P(t) w=0$, which follows from the arguments in [Rao 2001, Proposition 2.1], except that we replace the finite-dimensional $V$ by integrable $V$.

Now let $X_{\alpha}^{\prime} \otimes Q(t) t_{1} w=\lambda\left(X_{\alpha}^{\prime}, Q(t) t_{1}\right) w$ for $Q(t) \in I^{\prime}$. Then

$$
\left(X_{\alpha}^{\prime} \otimes Q(t) t_{1}\right)^{m} w=\lambda\left(X_{\alpha}^{\prime}, Q(t) t_{1}\right)^{m} w .
$$

Since $V$ is integrable, $\left(X_{\alpha}^{\prime} \otimes Q(t) t_{1}\right)^{m} w=0$. Hence $\lambda\left(X_{\alpha}^{\prime}, Q(t) t_{1}\right)^{m}=0$, and therefore $\lambda\left(X_{\alpha}^{\prime}, Q(t) t_{1}\right)=0$. This implies $X_{\alpha}^{\prime} \otimes Q(t) t_{1} w=0$. Similarly we can prove that $Y_{\alpha}^{\prime} \otimes Q(t) t_{1}^{-1} w=0$.

Claim 5.3b. $h_{\alpha}^{\prime} \otimes Q(t) w=0$.

Let $h_{\alpha}^{\prime} \otimes Q(t) w=\lambda\left(h_{\alpha}^{\prime}, Q(t)\right) w$ and let $\lambda=\lambda\left(h_{\alpha}^{\prime}, Q(t)\right)$.

Subclaim 1. $h_{\alpha}^{\prime} \otimes Q(t)\left(Y_{\alpha}^{\prime} \otimes t_{1}^{-1}\right)^{m} w=\lambda\left(Y_{\alpha}^{\prime} \otimes t_{1}^{-1}\right)^{m} w$. 
We prove this by induction on $m$. Let $m=1$ and consider $h_{\alpha}^{\prime} \otimes Q(t)\left(Y_{\alpha}^{\prime} \otimes t_{1}^{-1}\right) w=\left(Y_{\alpha}^{\prime} \otimes t_{1}^{-1}\right) h_{\alpha}^{\prime} \otimes Q(t) w-2 Y_{\alpha}^{\prime} \otimes t_{1}^{-1} Q(t) w=\lambda\left(Y_{\alpha}^{\prime} \otimes t_{1}^{-1}\right) w$.

Now we assume the claim for $m$ and consider

$$
\begin{aligned}
h_{\alpha}^{\prime} & \otimes Q(t)\left(Y_{\alpha}^{\prime} \otimes t_{1}^{-1}\right)^{m+1} w \\
& =\left(Y_{\alpha}^{\prime} \otimes t_{1}^{-1}\right)\left(h_{\alpha}^{\prime} \otimes Q(t)\right)\left(Y_{\alpha}^{\prime} \otimes t_{1}^{-1}\right)^{m} w+\left[h_{\alpha}^{\prime} \otimes Q(t), Y_{\alpha}^{\prime} \otimes t_{1}^{-1}\right]\left(Y_{\alpha}^{\prime} \otimes t_{1}^{-1}\right)^{m} w \\
& =\lambda\left(Y_{\alpha}^{\prime} \otimes t_{1}^{-1}\right)^{m+1} w-2 Y_{\alpha}^{\prime} \otimes Q(t) t_{1}^{-1}\left(Y_{\alpha}^{\prime} \otimes t_{1}^{-1}\right)^{m} w \\
& =\lambda\left(Y_{\alpha}^{\prime} \otimes t_{1}^{-1}\right)^{m+1} w,
\end{aligned}
$$

which follows by induction and the fact that $Y_{\alpha}^{\prime} \otimes Q(t) t_{1}^{-1} w=0$.

Subclaim 2. $X_{\alpha}^{\prime} \otimes Q(t) t_{1}\left(Y_{\alpha}^{\prime} \otimes t_{1}^{-1}\right)^{m} w=m \lambda\left(Y_{\alpha}^{\prime} \otimes t_{1}^{-1}\right)^{m-1} w$.

The proof is by induction on $m$. Let $m=1$. Then

$$
X_{\alpha}^{\prime} \otimes Q(t) t_{1}\left(Y_{\alpha}^{\prime} \otimes t_{1}^{-1}\right) w=Y_{\alpha}^{\prime} \otimes t_{1}^{-1} \cdot X_{\alpha}^{\prime} \otimes Q(t) t_{1} w+h_{\alpha}^{\prime} \otimes Q(t) w=\lambda w .
$$

Now assume the claim for $m$, and consider

$$
\begin{aligned}
\left(X_{\alpha}^{\prime} \otimes Q(t)\right. & \left.t_{1}\right)\left(Y_{\alpha}^{\prime} \otimes t_{1}^{-1}\right)^{m+1} w \\
= & \left(Y_{\alpha}^{\prime} \otimes t_{1}^{-1}\right)\left(X_{\alpha}^{\prime} \otimes Q(t) t_{1}\right)\left(Y_{\alpha}^{\prime} \otimes t_{1}^{-1}\right)^{m} w+h_{\alpha}^{\prime} \otimes Q(t)\left(Y_{\alpha}^{\prime} \otimes t_{1}^{-1}\right)^{m} w \\
= & m \lambda\left(Y_{\alpha}^{\prime} \otimes t_{1}^{-1}\right)^{m} w+\lambda\left(Y_{\alpha}^{\prime} \otimes t_{1}^{-1}\right)^{m} w \\
& =(m+1) \lambda\left(Y_{\alpha}^{\prime} \otimes t_{1}^{-1}\right)^{m} w .
\end{aligned}
$$

Since $V$ is integrable, there exists an $n_{0}>0$ such that $\left(Y_{\alpha}^{\prime} \otimes t_{1}^{-1}\right)^{n_{0}} w=0$, and $\left(Y_{\alpha}^{\prime} \otimes t_{1}^{-1}\right)^{n_{0}-1} w \neq 0$. Then

$$
\begin{aligned}
\lambda\left(Y_{\alpha}^{\prime} \otimes t_{1}^{-1}\right)^{n_{0}-1} w & =h_{\alpha}^{\prime} \otimes Q(t)\left(Y_{\alpha}^{\prime} \otimes t_{1}^{-1}\right)^{n_{0}-1} w \\
& =\left[X_{\alpha}^{\prime} \otimes Q(t) t_{1}, Y_{\alpha}^{\prime} \otimes t_{1}^{-1}\right]\left(Y_{\alpha}^{\prime} \otimes t_{1}^{-1}\right)^{n_{0}-1} w \\
& =-\left(n_{0}-1\right) \lambda\left(Y_{\alpha}^{\prime} \otimes t_{1}^{-1}\right)^{n_{0}-1} w .
\end{aligned}
$$

This implies that either $n_{0}=0$ or $\lambda=0$. But, by choice, $n_{0} \neq 0$, and hence $\lambda=0$. This proves that $h_{\alpha}^{\prime} \otimes Q(t) w=0$.

Claim 5.3c. $\left[X_{\alpha}, Y_{\alpha}^{\prime} \otimes t_{1}^{-1}\right] \otimes Q^{\prime}(t) w=0$ for $Q^{\prime}(t) \in I^{\prime}$.

Let $\left[X_{\alpha}, Y_{\alpha}^{\prime} \otimes t_{1}^{-1}\right] \otimes Q^{\prime}(t) w=\lambda w$.

Subclaim 3. $\left[X_{\alpha}, Y_{\alpha}^{\prime} \otimes t_{1}^{-1}\right] \otimes Q^{\prime}(t)\left(Y_{\alpha}^{\prime} \otimes t_{1}^{-1}\right)^{m} w=\lambda\left(Y_{\alpha}^{\prime} \otimes t_{1}^{-1}\right)^{m} w$. 
We prove this for $m=1$ :

$$
\begin{aligned}
{\left[X_{\alpha},\right.} & \left.Y_{\alpha}^{\prime} \otimes t_{1}^{-1}\right] \otimes Q^{\prime}(t)\left(Y_{\alpha}^{\prime} \otimes t_{1}^{-1}\right) w \\
& =\left(Y_{\alpha}^{\prime} \otimes t_{1}^{-1}\right)\left[X_{\alpha}, Y_{\alpha}^{\prime} \otimes t_{1}^{-1}\right] \otimes Q^{\prime}(t) w+\left[\left[X_{\alpha}, Y_{\alpha}^{\prime} \otimes t_{1}^{-1}\right] \otimes Q^{\prime}(t), Y_{\alpha}^{\prime} \otimes t_{1}^{-1}\right] \\
& =\lambda\left(Y_{\alpha}^{\prime} \otimes t_{1}^{-1}\right) w .
\end{aligned}
$$

This follows by the assumption and the fact that

$$
\left[\left[X_{\alpha}, Y_{\alpha}^{\prime} \otimes t_{1}^{-1}\right] \otimes Q^{\prime}(t), Y_{\alpha}^{\prime} \otimes t_{1}^{-1}\right]=0 .
$$

In fact $X_{\alpha} \in \mathfrak{g}_{0}$ and $Y_{\alpha}^{\prime} \in \mathfrak{g}_{1}$ implies that $\left[X_{\alpha}, Y_{\alpha}^{\prime} \otimes t_{1}^{-1}\right] \otimes Q^{\prime}(t) \in \mathfrak{g}_{1,0} \otimes t_{1}^{-1} Q^{\prime}(t)$. So $\left[\left[X_{\alpha}, Y_{\alpha}^{\prime} \otimes t_{1}^{-1}\right] \otimes Q^{\prime}(t), Y_{\alpha}^{\prime} \otimes t_{1}^{-1}\right] \in \mathfrak{g}_{0, \alpha} \otimes t_{1}^{-2} Q^{\prime}(t)$. Since $\operatorname{dim} \mathfrak{g}_{0, \alpha}=1$, we have $\left[\left[X_{\alpha}, Y_{\alpha}^{\prime} \otimes t_{1}^{-1}\right] \otimes Q^{\prime}(t), Y_{\alpha}^{\prime} \otimes t_{1}^{-1}\right] \in X_{\alpha} \otimes t_{1}^{-2} Q^{\prime}(t)=0$, because $X_{\alpha} \otimes I^{\prime}=0$. Now assume the claim for $m$ and consider

$$
\begin{aligned}
{\left[X_{\alpha}, Y_{\alpha}^{\prime} \otimes t_{1}^{-1}\right] \otimes } & Q^{\prime}(t)\left(Y_{\alpha}^{\prime} \otimes t_{1}^{-1}\right)^{m+1} w \\
= & \left(Y_{\alpha}^{\prime} \otimes t_{1}^{-1}\right)\left[X_{\alpha}, Y_{\alpha}^{\prime} \otimes t_{1}^{-1}\right] Q^{\prime}(t)\left(Y_{\alpha}^{\prime} \otimes t_{1}^{-1}\right)^{m} w \\
& \quad+\left[\left[X_{\alpha}, Y_{\alpha}^{\prime} \otimes t_{1}^{-1}\right] \otimes Q^{\prime}(t), Y_{\alpha}^{\prime} \otimes t_{1}^{-1}\right]\left(Y_{\alpha}^{\prime} \otimes t_{1}^{-1}\right)^{m} w \\
= & \lambda\left(Y_{\alpha}^{\prime} \otimes t_{1}^{-1}\right)^{m+1} w,
\end{aligned}
$$

because $\left[\left[X_{\alpha}, Y_{\alpha}^{\prime} \otimes t_{1}^{-1}\right] \otimes Q^{\prime}(t), Y_{\alpha}^{\prime} \otimes t_{1}^{-1}\right]=0$, as proved above.

Subclaim 4. $\left(X_{\alpha} \otimes Q^{\prime}(t)\right)\left(Y_{\alpha}^{\prime} \otimes t_{1}^{-1}\right)^{m} w=m \lambda\left(Y_{\alpha}^{\prime} \otimes t_{1}^{-1}\right)^{m-1} w$.

We prove this by induction on $m$. Let $m=1$, and consider

$X_{\alpha} \otimes Q^{\prime}(t)\left(Y_{\alpha}^{\prime} \otimes t_{1}^{-1}\right) w=Y_{\alpha}^{\prime} \otimes t_{1}^{-1} X_{\alpha} \otimes Q^{\prime}(t) w+\left[X_{\alpha}, Y_{\alpha}^{\prime} \otimes t_{1}^{-1}\right] \otimes Q^{\prime}(t) w=\lambda w$,

because $X_{\alpha} \otimes Q^{\prime}(t) w=0$.

Let Subclaim 4 be true for $m$ and consider

$$
\begin{aligned}
X_{\alpha} & \otimes Q^{\prime}(t)\left(Y_{\alpha}^{\prime} \otimes t_{1}^{-1}\right)^{m+1} w \\
& =\left(Y_{\alpha}^{\prime} \otimes t_{1}^{-1}\right)\left(X_{\alpha} \otimes Q^{\prime}(t)\right)\left(Y_{\alpha}^{\prime} \otimes t_{1}^{-1}\right)^{m} w+\left[X_{\alpha} \otimes Q^{\prime}(t), Y_{\alpha}^{\prime} \otimes t_{1}^{-1}\right]\left(Y_{\alpha}^{\prime} \otimes t_{1}^{-1}\right)^{m} w \\
& =m \lambda\left(Y_{\alpha}^{\prime} \otimes t_{1}^{-1}\right)^{m} w+\lambda\left(Y_{\alpha}^{\prime} \otimes t_{1}^{-1}\right)^{m} w \\
& =(m+1) \lambda\left(Y_{\alpha}^{\prime} \otimes t_{1}^{-1}\right)^{m} w
\end{aligned}
$$

Since $V$ is integrable, there exists an $n_{0}>0$ such that $\left(Y_{\alpha}^{\prime} \otimes t_{1}^{-1}\right)^{n_{0}-1} w \neq 0$ but $\left(Y_{\alpha}^{\prime} \otimes t_{1}^{-1}\right)^{n_{0}} w=0$. Now

$$
\begin{aligned}
\lambda\left(Y_{\alpha}^{\prime} \otimes t_{1}^{-1}\right)^{n_{0}-1} w= & {\left[X_{\alpha}, Y_{\alpha}^{\prime} \otimes t_{1}^{-1}\right] \otimes Q^{\prime}(t)\left(Y_{\alpha}^{\prime} \otimes t_{1}^{-1}\right)^{n_{0}-1} w } \\
= & X_{\alpha} \otimes Q^{\prime}(t)\left(Y_{\alpha}^{\prime} \otimes t_{1}^{-1}\right)\left(Y_{\alpha}^{\prime} \otimes t_{1}^{-1}\right)^{n_{0}-1} w \\
& \quad-\left(Y_{\alpha}^{\prime} \otimes t_{1}^{-1}\right) X_{\alpha} \otimes Q^{\prime}(t)\left(Y_{\alpha}^{\prime} \otimes t_{1}^{-1}\right)^{n_{0}-1} w \\
= & -\left(n_{0}-1\right) \lambda\left(Y_{\alpha}^{\prime} \otimes t_{1}^{-1}\right)^{n_{0}-1} w,
\end{aligned}
$$


which means either $n_{0}=0$ or $\lambda=0$. But by our choice $n_{0} \neq 0$, and hence $\lambda=0$. This proves Claim 5.3c. Similarly we can prove that $\left[X_{\alpha}^{\prime} \otimes t_{1}, Y_{\alpha}\right] \otimes Q^{\prime}(t) w=0$ for $Q^{\prime}(t) \in I^{\prime}$.

Now consider the nonzero submodule $W=\left\{w \in V(\psi) \mid G_{\alpha} w=0\right\}$ of $V(\psi)$. Since $V(\psi)$ is irreducible, we have $W=V(\psi)$. Thus the module $V(\psi)$ is zero on $I^{\prime}$. Let

$$
I^{\prime \prime}=\left\langle P_{1}\left(t_{1}^{2}\right)=t_{1} \prod_{i=1}^{N_{1}}\left(t_{1}^{2}-a_{1 i}^{2}\right), \quad P_{j}\left(t_{j}\right)=\prod_{l=1}^{N_{j}}\left(t_{j}-a_{j l}\right) \quad \text { for } 2 \leq j \leq(n-1)\right\rangle .
$$

In a similar way as above, we can prove that the module $V(\psi)$ is zero on $I^{\prime \prime}$. So the module $V(\psi)$ is zero on kernel $\phi(\mu)$ by Lemma 4.2. Thus $V(\psi)$ is a module for $\bigoplus_{N} \mathfrak{g}_{\text {aff }}$.

\section{When the diagram automorphism is of order 3}

We now consider the case when $\mu$ is a diagram automorphism of $\mathfrak{g}$ of order 3 . We will prove analogues of Proposition 4.1 and Lemma 4.2 in this case.

In this case also, $\mu$ leaves $\mathfrak{h}$ invariant. Let $\mathfrak{h}(\mu)$ be the $\mu$-fixed points of $\mathfrak{h}$, and let $\epsilon$ be a primitive third root of unity. In this case $\mathfrak{g}=\mathfrak{g}_{0} \oplus \mathfrak{g}_{1} \oplus \mathfrak{g}_{-1}$, where $\mathfrak{g}_{i}$ is the eigenspace of $\mu$ for the eigenvalue $\epsilon^{i}$ for $i \in\{0,1,-1\}$. Suppose $(X, Y) \neq 0$ implies $X, Y \in \mathfrak{g}_{0}$ or $X \in \mathfrak{g}_{1}$ and $Y \in \mathfrak{g}_{-1}$. Because of this, the center in the twisted toroidal Lie algebra, which we denote by $\left(\Omega_{A} / d_{A}\right)^{0}$ will be

$$
\left(\Omega_{A} / d_{A}\right)^{0}=\operatorname{span}\left\{t^{\underline{m}} K_{i} \mid m_{i_{1}}+\cdots+m_{i_{k}} \cong 0(\bmod 3)\right\} .
$$

Also $\mu$ leaves $\mathfrak{g} \otimes A$ invariant. Let $(\mathfrak{g} \otimes A)_{\mu}$ be the $\mu$-fixed points of $\mathfrak{g} \otimes A$. Then $\tau(\mu)=(\mathfrak{g} \otimes A)_{\mu} \oplus\left(\Omega_{A} / d_{A}\right)^{0} \oplus D$. It is known from [Berman and Krylyuk 1995] that $(\mathfrak{g} \otimes A)_{\mu} \oplus\left(\Omega_{A} / d_{A}\right)^{0}$ is the universal central extension of $(\mathfrak{g} \otimes A)_{\mu}$. In this case also we choose the coordinates in such a way that $S=\{1, n\}$. Here

$$
\begin{aligned}
& \tau(\mu)=\bigoplus_{m \in \mathbb{Z}} \mathfrak{g}_{0} \otimes t_{n}^{3 m} t_{1}^{3 m_{1}} t_{2}^{m_{2}} \cdots t_{n-1}^{m_{n-1}} \\
& \bigoplus \mathfrak{g}_{0} \otimes t_{n}^{3 m+1} t_{1}^{3 m_{1}+2} t_{2}^{m_{2}} \cdots t_{n-1}^{m_{n-1}} \\
& \bigoplus \mathfrak{g}_{0} \otimes t_{n}^{3 m+2} t_{1}^{3 m_{1}+1} t_{2}^{m_{2}} \cdots t_{n-1}^{m_{n-1}} \\
& m \in \mathbb{Z} \\
& \bigoplus \mathfrak{g}_{1} \otimes t_{n}^{3 m+1} t_{1}^{3 m_{1}+1} t_{2}^{m_{2}} \cdots t_{n-1}^{m_{n-1}} \\
& m \in \mathbb{Z} \\
& \bigoplus \mathfrak{g}_{-1} \otimes t_{n}^{3 m} t_{1}^{3 m_{1}+1} t_{2}^{m_{2}} \cdots t_{n-1}^{m_{n-1}} \\
& m \in \mathbb{Z} \\
& m \in \mathbb{Z} \\
& \bigoplus \mathfrak{g}_{1} \otimes t_{n}^{3 m} t_{1}^{3 m_{1}+2} t_{2}^{m_{2}} \cdots t_{n-1}^{m_{n-1}} \\
& m \in \mathbb{Z} \\
& \bigoplus \mathfrak{g}_{-1} \otimes t_{n}^{3 m+2} t_{1}^{3 m_{1}+2} t_{2}^{m_{2}} \cdots t_{n-1}^{m_{n-1}} \\
& \bigoplus \mathfrak{g}_{1} \otimes t_{n}^{3 m+2} t_{1}^{3 m_{1}} t_{2}^{m_{2}} \cdots t_{n-1}^{m_{n-1}} \\
& m \in \mathbb{Z} \\
& m \in \mathbb{Z} \\
& \bigoplus \mathfrak{g}_{-1} \otimes t_{n}^{3 m+1} t_{1}^{3 m_{1}} t_{2}^{m_{2}} \cdots t_{n-1}^{m_{n-1}} \\
& m \in \mathbb{Z} \\
& \bigoplus\left(\Omega_{A} / d_{A}\right)^{0} \bigoplus D
\end{aligned}
$$


Also let

$$
\begin{aligned}
& \tau_{0}(\mu)=\bigoplus_{m \in \mathbb{Z}} \mathfrak{g}_{0} \otimes t_{n}^{3 m} t_{1}^{3 m_{1}} t_{2}^{m_{2}} \cdots t_{n-1}^{m_{n-1}} \\
& \bigoplus \mathfrak{g}_{0} \otimes t_{n}^{3 m+1} t_{1}^{3 m_{1}+2} t_{2}^{m_{2}} \cdots t_{n-1}^{m_{n-1}} \\
& \bigoplus \mathfrak{g}_{0} \otimes t_{n}^{3 m+2} t_{1}^{3 m_{1}+1} t_{2}^{m_{2}} \cdots t_{n-1}^{m_{n-1}} \\
& m \in \mathbb{Z} \\
& \bigoplus \mathfrak{g}_{1} \otimes t_{n}^{3 m+1} t_{1}^{3 m_{1}+1} t_{2}^{m_{2}} \cdots t_{n-1}^{m_{n-1}} \\
& m \in \mathbb{Z} \\
& \bigoplus \mathfrak{g}_{-1} \otimes t_{n}^{3 m} t_{1}^{3 m_{1}+1} t_{2}^{m_{2}} \cdots t_{n-1}^{m_{n-1}} \\
& m \in \mathbb{Z} \\
& m \in \mathbb{Z} \\
& \bigoplus \mathfrak{g}_{1} \otimes t_{n}^{3 m} t_{1}^{3 m_{1}+2} t_{2}^{m_{2}} \cdots t_{n-1}^{m_{n-1}} \\
& m \in \mathbb{Z} \\
& \bigoplus \mathfrak{g}_{1} \otimes t_{n}^{3 m+2} t_{1}^{3 m_{1}} t_{2}^{m_{2}} \cdots t_{n-1}^{m_{n-1}} \\
& m \in \mathbb{Z} \\
& \bigoplus \mathfrak{g}_{-1} \otimes t_{n}^{3 m+2} t_{1}^{3 m_{1}+2} t_{2}^{m_{2}} \cdots t_{n-1}^{m_{n-1}} \bigoplus\left(\Omega_{A} / d_{A}\right)^{0} . \\
& m \in \mathbb{Z} \\
& \bigoplus \mathfrak{g}_{-1} \otimes t_{n}^{3 m+1} t_{1}^{3 m_{1}} t_{2}^{m_{2}} \cdots t_{n-1}^{m_{n-1}} \\
& m \in \mathbb{Z}
\end{aligned}
$$

Root system for $\tau(\mu)$. Let $m=3$. Then

$$
\begin{aligned}
& \tau(\mu)=\mathfrak{g}_{0} \otimes \sum_{m_{i_{1}}+\cdots+m_{i_{k}}} \cong 0(\bmod 3) \mathbb{C} t^{\underline{m}} \bigoplus \mathfrak{g}_{1} \otimes \sum_{m_{i_{1}}+\cdots+m_{i_{k}}} \cong 1(\bmod 3) \mathbb{C} t^{\underline{m}} \\
& \\
& \bigoplus \mathfrak{g}_{-1} \otimes \sum_{m_{i_{1}}+\cdots+m_{i_{k}}} \cong 1(\bmod 3) \mathbb{C} t^{\underline{m}} \bigoplus\left(\Omega_{A} / d_{A}\right)^{0} \bigoplus D .
\end{aligned}
$$

Let $\Delta_{0}$ be the roots of $\mathfrak{g}_{0}$ and $\Delta_{0}^{s}$ be the short roots of $\mathfrak{g}_{0}$. Define $\Delta_{\mathrm{re}}=\left\{\alpha+\delta_{\underline{m}} \mid \alpha \in \Delta_{0}, m_{i_{1}}+\cdots+m_{i_{k}} \cong 0(\bmod 3)\right\}$

$$
\bigcup\left\{\alpha+\delta_{\underline{m}} \mid \alpha \in \Delta_{0}^{s}, m_{i_{1}}+\cdots+m_{i_{k}} \cong 1(\bmod 3)\right\},
$$

and let $\Delta_{\mathrm{im}}=\left\{\delta_{\underline{m}} \mid \underline{m} \in \mathbb{Z}^{n}\right\}$. Let $\Delta=\Delta_{\mathrm{re}} \cup \Delta_{\mathrm{im}}$.

Define

$$
\Delta_{\mathrm{re}}^{+}=\left\{\alpha+\delta_{\underline{m}} \mid \alpha>0, m_{n} \geq 0 \text { or } \alpha<0, m_{n} \geq 1\right\}, \quad \Delta_{\mathrm{im}}^{+}=\left\{\delta_{\underline{m}} \mid m_{n} \geq 1\right\},
$$

and $\Delta^{0}=\left\{\delta_{m} \mid m_{n}=0\right\}$. Then $\Delta^{+}=\Delta_{\text {re }}^{+} \cup \Delta_{\text {im }}^{+}$and $\Delta^{-}=-\Delta^{+}$. Further $\Delta=\Delta^{+} \cup \Delta^{0} \cup \Delta^{-}$. Let $\alpha \in \Delta$. Then $\alpha \in \Delta_{\text {re }}$ if and only if $\langle\alpha, \alpha\rangle \neq 0$.

Let $n \geq 2$ be a positive integer. For each $i$ in $1 \leq i \leq(n-1)$, let $N_{i}$ be a positive integer. Let $\underline{a}_{i}=\left(a_{i 1}, a_{i 2}, \ldots, a_{i N_{i}}\right)$ be nonzero distinct complex numbers. Let $I=\left(i_{1}, i_{2}, \ldots, i_{n-1}\right)$, where $1 \leq i_{j} \leq N_{j}$. Let $\underline{m}^{\prime}=\left(m_{1}, m_{2}, \ldots, m_{n-1}\right) \in \mathbb{Z}^{n-1}$. Let $\mathfrak{g}_{\text {aff }}=\mathfrak{g} \otimes \mathbb{C}\left[t_{n}, t_{n}^{-1}\right] \oplus \mathbb{C} K_{n}$. Let $\phi$ be the Lie algebra homomorphism

$$
\begin{aligned}
\phi: \mathfrak{g}_{\mathrm{aff}} \otimes \mathbb{C}\left[t_{1}^{ \pm 1}, t_{2}^{ \pm 1}, \ldots, t_{n-1}^{ \pm 1}\right] & \rightarrow\left(\bigoplus_{N=N_{1} N_{2} \cdots N_{n-1} \text {-copies }} \mathfrak{g}_{\mathrm{aff}}\right)=\mathfrak{g}_{\mathrm{aff} N}, \\
X t_{1}^{m_{1}} \cdots t_{n-1}^{m_{n-1}} & \mapsto\left(a_{i_{1} m_{1}}^{m_{2 i_{2}}} \cdots a_{(n-1) i_{n-1}}^{m_{n-1}} X\right)_{1 \leq i_{1} \leq N_{1} ; \ldots ; 1 \leq i_{n-1} \leq N_{n-1}},
\end{aligned}
$$

where $X \in \mathfrak{g}_{\text {aff }}$.

Following the proof of [Rao 2004, Lemma 3.11(a)], we see that $\phi$ is surjective. Let $T \subset\left\{11,12, \ldots, 1 N_{1}\right\}$ be a maximal set such that $a_{1 i}^{3} \neq a_{1 j}^{3}$ whenever $1 i, 1 j \in T$ and $1 \leq i, j \leq N_{1}$. 
Proposition 6.1. Let $\phi(\mu)=\phi \mid \tau_{0}(\mu)$. If the cardinality of $T$ is $N_{1}$, then $\phi(\mu)$ is a surjective Lie algebra homomorphism from $\tau_{0}(\mu)$ to $\mathfrak{g}_{\mathrm{aff}}{ }_{N}$.

Proof. We know that if $m=3$, then $\mathfrak{g}=\mathfrak{g}_{0} \oplus \mathfrak{g}_{1} \oplus \mathfrak{g}_{-1}$. Now there are ten cases.

Case 6.1a. We fix $m_{n} \in \mathbb{Z}$, and let $Y_{1}, Y_{2}, \ldots, Y_{N} \in \mathfrak{g}_{0}$. Let

$$
\phi(\mu)\left(\sum_{i=1}^{N} b_{i} X_{i} t_{n}^{3 m_{n}} t_{1}^{3 m_{1}} t_{2}^{m_{2}} \cdots t_{n-1}^{m_{n-1}}\right)=\left(Y_{1} t_{n}^{3 m_{n}}, Y_{2} t_{n}^{3 m_{n}}, \ldots, Y_{N} t_{n}^{3 m_{n}}\right),
$$

where the $b_{i}$ are complex numbers and $X_{i} t_{n}^{3 m_{n}} t_{1}^{3 m_{1}} t_{2}^{m_{2}} \cdots t_{n-1}^{m_{n-1}} \in \tau_{0}(\mu)$ and $0 \leq$ $m_{j} \leq N_{j}-1$ for $1 \leq j \leq(n-1)$. So, we must solve the equation

$$
\left(\sum_{i=1}^{N} b_{i} X_{i} t_{n}^{3 m_{n}} a_{1 i_{1}}^{3 m_{1}} a_{2 i_{2}}^{m_{2}} \cdots a_{(n-1) i_{n-1}}^{m_{n-1}}\right)=\left(Y_{1} t_{n}^{3 m_{n}}, Y_{2} t_{n}^{3 m_{n}}, \ldots, Y_{N} t_{n}^{3 m_{n}}\right) .
$$

Writing this in matrix form, we must prove that the matrix

$$
M=\left(a_{1 i_{1}}^{3 m_{1}} a_{2 i_{2}}^{m_{2}} \cdots a_{(n-1) i_{n-1}}^{m_{n-1}}\right)
$$

is invertible, where $\left(i_{1}, i_{2}, \ldots, i_{n-1}\right)$ for $1 \leq i_{j} \leq N_{j}$ determines the rows and $\underline{m}^{\prime}=\left(m_{1}, m_{2}, \ldots, m_{n-1}\right)$ where $0 \leq m_{j} \leq N_{j}-1$ for all $1 \leq j \leq(n-1)$, determines the columns. The matrix $M$ is a tensor product of other matrices:

$$
M=\left(a_{1 i_{1}}^{3 m_{1}}\right)_{1 \leq i_{1} \leq N_{1}, 0 \leq m_{1} \leq N_{1}-1} \otimes M_{2} \otimes \cdots \otimes M_{n-1},
$$

where the $M_{j}$ for $2 \leq j \leq(n-1)$ defined as in Equation (1). Let

$$
M_{1}=\left(a_{1 i_{1}}^{3 m_{1}}\right)_{1 \leq i_{1} \leq N_{1}, 0 \leq m_{1} \leq N_{1}-1} .
$$

If the cardinality of $T$ is $N_{1}$, which means the $a_{1 i_{1}}^{3}$ are all distinct for all $1 \leq i_{1} \leq N_{1}$, then $M_{1}$ is a Vandermonde matrix and is therefore invertible. Similarly $M_{2}$ is a Vandermonde matrix because $a_{2 i} \neq a_{2 j}$ for all $1 \leq i, j \leq N_{2}$ with $i \neq j$. Similarly $M_{j}$ is also invertible for $3 \leq j \leq(n-1)$. According to a standard result of linear algebra, the determinant of a tensor product of invertible matrices is nonzero. So matrix $M$ is invertible.

Case 6.1b. We fix $m_{n} \in \mathbb{Z}$, and let $Y_{1}, Y_{2}, \ldots, Y_{N} \in \mathfrak{g}_{0}$. Let $\phi(\mu)\left(\sum_{i=1}^{N} b_{i} X_{i} t_{n}^{3 m_{n}+1} t_{1}^{3 m_{1}+2} t_{2}^{m_{2}} \cdots t_{n-1}^{m_{n-1}}\right)=\left(Y_{1} t_{n}^{3 m_{n}+1}, Y_{2} t_{n}^{3 m_{n}+1}, \ldots, Y_{N} t_{n}^{3 m_{n}+1}\right)$, 
where the $b_{i}$ are complex numbers, $X_{i} t_{n}^{3 m_{n}+1} t_{1}^{3 m_{1}+2} t_{2}^{m_{2}} \cdots t_{n-1}^{m_{n-1}} \in \tau_{0}(\mu)$, and $0 \leq$ $m_{j} \leq N_{j}-1$ for $1 \leq j \leq(n-1)$. So, we must solve the equation

$$
\left(\sum_{i=1}^{N} b_{i} X_{i} t_{n}^{3 m_{n}+1} a_{1 i_{1}}^{3 m_{1}+2} a_{2 i_{2}}^{m_{2}} \cdots a_{(n-1) i_{n-1}}^{m_{n-1}}\right)=\left(Y_{1} t_{n}^{3 m_{n}+1}, Y_{2} t_{n}^{3 m_{n}+1}, \ldots, Y_{N} t_{n}^{3 m_{n}+1}\right) .
$$

Writing this in matrix form, we must prove that the matrix

$$
M=\left(a_{1 i_{1}}^{3 m_{1}+2} a_{2 i_{2}}^{m_{2}} \cdots a_{(n-1) i_{n-1}}^{m_{n-1}}\right)
$$

is invertible, where $\left(i_{1}, i_{2}, \ldots, i_{n-1}\right)$ for $1 \leq i_{j} \leq N_{j}$ determines the rows and $\underline{m}^{\prime}=\left(m_{1}, m_{2}, \ldots, m_{n-1}\right)$, where $0 \leq m_{j} \leq N_{j}-1$ for all $1 \leq j \leq(n-1)$, determines the columns. The matrix $M$ is a tensor product of other matrices

$$
M=\left(a_{1 i_{1}}^{3 m_{1}+2}\right)_{1 \leq i_{1} \leq N_{1}, 0 \leq m_{1} \leq N_{1}-1} \otimes M_{2} \otimes \cdots \otimes M_{n-1} .
$$

Let $M_{1}=\left(a_{1 i_{1}}^{3 m_{1}+2}\right)_{1 \leq i_{1} \leq N_{1}, 0 \leq m_{1} \leq N_{1}-1}$. Again, as above if the cardinality of $T$ is $N_{1}$, then $M_{1}$ is product of two matrices: one is the diagonal matrix whose diagonal entries are $a_{11}^{2}, a_{12}^{2}, \ldots, a_{1 N_{1}}^{2}$, and the other is $\left(a_{1 i_{1}}^{3 m_{1}}\right)_{1 \leq i_{1} \leq N_{1}, 0 \leq m_{1} \leq N_{1}-1}$. Since $a_{11} \neq a_{12} \neq \cdots \neq a_{1 N_{1}}$, we have $a_{11}^{2} \neq a_{12}^{2} \neq \cdots \neq a_{1 N_{1}}^{2}$. Hence the diagonal matrix is invertible. The other matrix is a Vandermonde matrix and hence is invertible. So $M_{1}$ is invertible. Similarly the $M_{j}$ for $2 \leq j \leq(n-1)$ are invertible since they are Vandermonde matrices. Hence $M$ is invertible.

Case 6.1c. We fix $m_{n} \in \mathbb{Z}$, and let $Y_{1}, Y_{2}, \ldots, Y_{N} \in \mathfrak{g}_{0}$. Let

$\phi(\mu)\left(\sum_{i=1}^{N} b_{i} X_{i} t_{n}^{3 m_{n}+2} t_{1}^{3 m_{1}+1} t_{2}^{m_{2}} \cdots t_{n-1}^{m_{n-1}}\right)=\left(Y_{1} t_{n}^{3 m_{n}+2}, Y_{2} t_{n}^{3 m_{n}+2}, \ldots, Y_{N} t_{n}^{3 m_{n}+2}\right)$,

where the $b_{i}$ are complex numbers, $X_{i} t_{n}^{3 m_{n}+2} t_{1}^{3 m_{1}+1} t_{2}^{m_{2}} \cdots t_{n-1}^{m_{n-1}} \in \tau_{0}(\mu)$, and $0 \leq$ $m_{j} \leq N_{j}-1$ for $1 \leq j \leq(n-1)$. So, solving the equation

$$
\left(\sum_{i=1}^{N} b_{i} X_{i} t_{n}^{3 m_{n}+2} a_{1 i_{1}}^{3 m_{1}+1} a_{2 i_{2}}^{m_{2}} \cdots a_{(n-1) i_{n-1}}^{m_{n-1}}\right)=\left(Y_{1} t_{n}^{3 m_{n}+2}, Y_{2} t_{n}^{3 m_{n}+2}, \ldots, Y_{N} t_{n}^{3 m_{n}+2}\right),
$$

we must prove that the matrix

$$
M=\left(a_{1 i_{1}}^{3 m_{1}+1}\right)_{1 \leq i_{1} \leq N_{1}, 0 \leq m_{1} \leq N_{1}-1} \otimes M_{2} \otimes \cdots \otimes M_{n-1},
$$

is invertible. Let $M_{1}=\left(a_{1 i_{1}}^{3 m_{1}+1}\right)_{1 \leq i_{1} \leq N_{1}, 0 \leq m_{1} \leq N_{1}-1}$. Again, if the cardinality of $T$ is $N_{1}$, then $M_{1}$ is product of two matrices: one is the diagonal matrix whose diagonal entries are $a_{11}, a_{12}, \ldots, a_{1 N_{1}}$, and the other is $\left(a_{1 i_{1}}^{3 m_{1}}\right)_{1 \leq i_{1} \leq N_{1}, 0 \leq m_{1} \leq N_{1}-1}$. Since $a_{11} \neq a_{12} \neq \cdots \neq a_{1 N_{1}}$, the diagonal matrix is invertible. And the other matrix is a Vandermonde matrix and hence invertible. So $M_{1}$ is invertible. Similarly the 
$M_{j}$ for $2 \leq j \leq(n-1)$ are invertible as they are Vandermonde matrices. Hence $M$ is invertible.

Case 6.1d. If we consider $\left(X_{i} t_{n}^{3 m_{n}} t_{1}^{3 m_{1}+2} t_{2}^{m_{2}} \cdots t_{n-1}^{m_{n-1}}\right) \in \tau_{0}(\mu)$, where $X_{i} \in \mathfrak{g}_{1}$, then the proof of this case is similar to that of Case 6.1b.

Case 6.1e. If we consider $\left(X_{i} t_{n}^{3 m_{n}+1} t_{1}^{3 m_{1}+1} t_{2}^{m_{2}} \cdots t_{n-1}^{m_{n-1}}\right) \in \tau_{0}(\mu)$, where $X_{i} \in \mathfrak{g}_{1}$, then the proof of this case is similar to that of Case 6.1c.

Case 6.1f. If we consider $\left(X_{i} t_{n}^{3 m_{n}+2} t_{1}^{3 m_{1}} t_{2}^{m_{2}} \cdots t_{n-1}^{m_{n-1}}\right) \in \tau_{0}(\mu)$, where $X_{i} \in \mathfrak{g}_{1}$, then the proof of this case is similar to that of Case 6.1a.

Case 6.1g. If we consider $\left(X_{i} t_{n}^{3 m_{n}} t_{1}^{3 m_{1}+1} t_{2}^{m_{2}} \cdots t_{n-1}^{m_{n-1}}\right) \in \tau_{0}(\mu)$, where $X_{i} \in \mathfrak{g}_{-1}$, then the proof of this case is similar to that of Case 6.1c.

Case 6.1h. If we consider $\left(X_{i} t_{n}^{3 m_{n}+1} t_{1}^{3 m_{1}} t_{2}^{m_{2}} \cdots t_{n-1}^{m_{n-1}}\right) \in \tau_{0}(\mu)$, where $X_{i} \in \mathfrak{g}_{-1}$, then the proof of this case is similar to that of Case 6.1a.

Case 6.1i. If we consider $\left(X_{i} t_{n}^{3 m_{n}+2} t_{1}^{3 m_{1}+2} t_{2}^{m_{2}} \cdots t_{n-1}^{m_{n-1}}\right) \in \tau_{0}(\mu)$, where $X_{i} \in \mathfrak{g}_{-1}$, then the proof of this case is similar to that of Case $6.1 \mathrm{~b}$.

Case 6.1j. Let $c_{1}, c_{2}, \ldots, c_{N} \in \mathbb{C}$. Let

$$
\phi(\mu)\left(\sum_{i=1}^{N} b_{i} K_{n} t_{1}^{3 m_{1}} t_{2}^{m_{2}} \cdots t_{n-1}^{m_{n-1}}\right)=\left(c_{1} K_{n}, c_{2} K_{n}, \ldots, c_{N} K_{n}\right),
$$

where the $b_{i}$ are complex numbers, $K_{n} t_{1}^{3 m_{1}} t_{2}^{m_{2}} \cdots t_{n-1}^{m_{n-1}} \in \tau_{0}(\mu)$, and $0 \leq m_{j} \leq$ $N_{j}-1$ for $1 \leq j \leq(n-1)$. So, solving the equation

$$
\left(\sum_{i=1}^{N} b_{i} K_{n} a_{1 i_{1}}^{3 m_{1}} a_{2 i_{2}}^{m_{2}} \cdots a_{(n-1) i_{n-1}}^{m_{n-1}}\right)=\left(c_{1} K_{n}, c_{2} K_{n}, \ldots, c_{N} K_{n}\right),
$$

we must prove that the matrix

$$
M=\left(a_{1 i_{1}}^{3 m_{1}} a_{2 i_{2}}^{m_{2}} \cdots a_{(n-1) i_{n-1}}^{m_{n-1}}\right)
$$

is invertible, where $\left(i_{1}, i_{2}, \ldots, i_{n-1}\right)$ for $1 \leq i_{j} \leq N_{j}$ determines the rows and $\underline{m}^{\prime}=\left(m_{1}, m_{2}, \ldots, m_{n-1}\right)$, where $0 \leq m_{j} \leq N_{j}-1$ for all $1 \leq j \leq(n-1)$, determines the columns. Again by Case 6.1a, $M$ is invertible if the cardinality of $T$ is $N_{1}$.

So if the cardinality of $T$ is $N_{1}$, then $\phi(\mu)$ is a surjective Lie algebra homomorphism from $\tau_{0}(\mu)$ to $\mathfrak{g}_{\text {aff }}{ }_{N}$.

We now define polynomials $P_{1}\left(t_{1}^{3}\right)$ and $P_{2}\left(t_{2}\right), \ldots, P_{n-1}\left(t_{n-1}\right)$ by

$$
P_{1}\left(t_{1}^{3}\right)=\prod_{i=1}^{N_{1}}\left(t_{1}^{3}-a_{1 i}^{3}\right) \quad \text { and } \quad P_{j}\left(t_{j}\right)=\prod_{l=1}^{N_{j}}\left(t_{j}-a_{j l}\right) \quad \text { for } 2 \leq j \leq(n-1) .
$$


Notation $\left\langle P_{1}\left(t_{1}^{3}\right), P_{2}\left(t_{2}\right), \ldots, P_{n-1}\left(t_{n-1}\right)\right\rangle$ denotes the ideal generated by the polynomials $P_{1}\left(t_{1}^{3}\right), P_{2}\left(t_{2}\right), \ldots, P_{n-1}\left(t_{n-1}\right)$ inside $\mathbb{C}\left[t_{1}^{ \pm 3}, t_{2}^{ \pm 1}, \ldots, t_{n-1}^{ \pm 1}\right]$.

Lemma 6.2. If the cardinality of $T$ is $N_{1}$, then $\operatorname{ker} \phi(\mu)$ is given by $I_{1}+I_{2}+I_{3}+$ $I_{4}+I_{5}+I_{6}+I_{7}+I_{8}+I_{9}+I_{10}$, where for some fixed $m \in \mathbb{Z}$,

$$
\begin{aligned}
& I_{1}=\mathfrak{g}_{0} \otimes t_{n}^{3 m} \quad\left\langle P_{1}\left(t_{1}^{3}\right), P_{2}\left(t_{2}\right), \ldots, P_{n-1}\left(t_{n-1}\right)\right\rangle, \\
& I_{2}=\mathfrak{g}_{0} \otimes t_{n}^{3 m+1}\left\langle t_{1}^{2} P_{1}\left(t_{1}^{3}\right), P_{2}\left(t_{2}\right), \ldots, P_{n-1}\left(t_{n-1}\right)\right\rangle, \\
& I_{3}=\mathfrak{g}_{0} \otimes t_{n}^{3 m+2}\left\langle t_{1} P_{1}\left(t_{1}^{3}\right), P_{2}\left(t_{2}\right), \ldots, P_{n-1}\left(t_{n-1}\right)\right\rangle, \\
& I_{4}=\mathfrak{g}_{1} \otimes t_{n}^{3 m} \quad\left\langle t_{1}^{2} P_{1}\left(t_{1}^{3}\right), P_{2}\left(t_{2}\right), \ldots, P_{n-1}\left(t_{n-1}\right)\right\rangle, \\
& I_{5}=\mathfrak{g}_{1} \otimes t_{n}^{3 m+1}\left\langle t_{1} P_{1}\left(t_{1}^{3}\right), P_{2}\left(t_{2}\right), \ldots, P_{n-1}\left(t_{n-1}\right)\right\rangle, \\
& I_{6}=\mathfrak{g}_{1} \otimes t_{n}^{3 m+2}\left\langle P_{1}\left(t_{1}^{3}\right), P_{2}\left(t_{2}\right), \ldots, P_{n-1}\left(t_{n-1}\right)\right\rangle \text {, } \\
& I_{7}=\mathfrak{g}_{-1} \otimes t_{n}^{3 m} \quad\left\langle t_{1} P_{1}\left(t_{1}^{3}\right), P_{2}\left(t_{2}\right), \ldots, P_{n-1}\left(t_{n-1}\right)\right\rangle, \\
& I_{8}=\mathfrak{g}_{-1} \otimes t_{n}^{3 m+1}\left\langle P_{1}\left(t_{1}^{3}\right), P_{2}\left(t_{2}\right), \ldots, P_{n-1}\left(t_{n-1}\right)\right\rangle \text {, } \\
& I_{9}=\mathfrak{g}_{-1} \otimes t_{n}^{3 m+2}\left\langle t_{1}^{2} P_{1}\left(t_{1}^{3}\right), P_{2}\left(t_{2}\right), \ldots, P_{n-1}\left(t_{n-1}\right)\right\rangle, \\
& I_{10}=K_{n} \otimes \quad\left\langle P_{1}\left(t_{1}^{3}\right), P_{2}\left(t_{2}\right), \ldots, P_{n-1}\left(t_{n-1}\right)\right\rangle .
\end{aligned}
$$

Proof. We consider the map $\phi_{1}$ defined by

$$
\begin{aligned}
\mathfrak{g}_{0} \otimes t_{n}^{3 m} t_{1}^{3 m_{1}} t_{2}^{m_{2}} \cdots t_{n-1}^{m_{n-1}} & \rightarrow \bigoplus_{N=N_{1} N_{2} \cdots N_{n-1}} \mathfrak{g}_{0} \otimes t_{n}^{3 m}, \\
X t_{n}^{3 m} t_{1}^{3 m_{1}} t_{2}^{m_{2}} \cdots t_{n-1}^{m_{n-1}} & \mapsto\left(X t_{n}^{3 m} a_{1 i_{1}}^{3 m_{1}} a_{2 i_{2}}^{m_{2}} \cdots a_{(n-1) i_{n-1}}^{m_{n-1}}\right)_{1 \leq i_{1} \leq N_{1} ; \ldots ; 1 \leq i_{n-1} \leq N_{n-1}} .
\end{aligned}
$$

Let $M=\left(N=N_{1} N_{2} \cdots N_{n-1}\right)$ (the dimension of $\left.\mathfrak{g}_{0}\right)$. As in Proposition 6.1, one can show that $\phi_{1}$ is surjective. Since $\phi_{1}$ is nothing but the evaluation map at the roots of $P_{1}\left(t_{1}^{3}\right), P_{2}\left(t_{2}\right), \ldots, P_{n-1}\left(t_{n-1}\right)$, we have $I_{1} \subset \operatorname{ker} \phi_{1}$. So

$$
\operatorname{dim}\left(\mathfrak{g}_{0} \otimes t_{n}^{3 m} t_{1}^{3 m_{1}} t_{2}^{m_{2}} \cdots t_{n-1}^{m_{n-1}} / I_{1}\right) \geq M .
$$

Consider the space

$$
R=\left\{\mathfrak{g}_{0} \otimes t_{n}^{3 m} t_{1}^{3 m_{1}} t_{2}^{m_{2}} \cdots t_{n-1}^{m_{n-1}} \mid 0 \leq m_{i} \leq N_{i}-1 \text { for } 1 \leq i \leq(n-1)\right\} .
$$

Any element of $\mathfrak{g}_{0} \otimes t_{n}^{3 m} t_{1}^{3 m_{1}} t_{2}^{m_{2}} \cdots t_{n-1}^{m_{n-1}}$ can be written as linear combination of elements of $R$ modulo $I_{1}$. Hence $\phi_{1}$ is injective on $R$ modulo $I_{1}$, since the corresponding matrix is invertible. So $R$ is a spanning set for

$$
\left(\mathfrak{g}_{0} \otimes t_{n}^{3 m} t_{1}^{3 m_{1}} t_{2}^{m_{2}} \cdots t_{n-1}^{m_{n-1}} / I_{1}\right),
$$

and $\operatorname{dim}\left(\mathfrak{g}_{0} \otimes t_{n}^{3 m} t_{1}^{3 m_{1}} t_{2}^{m_{2}} \cdots t_{n-1}^{m_{n-1}} / I_{1}\right) \leq M$. So $I_{1}$ is the kernel of $\phi_{1}$. Similarly arguing on the other components of $\tau_{0}(\mu)$, we get that $\sum_{j=1}^{10} I_{j}$ is the kernel of $\phi(\mu)$ if the cardinality of $T$ is $N_{1}$. This proves Lemma 6.2. 
Remark 6.3. The results of Section 5 can be proved similarly in case $m=3$.

Note 6.4. The proof of Proposition 2.6 will remain same in case $m=3$ except that for $\lambda, \lambda+\delta_{1}, \lambda+2 \delta_{1}$ or $\lambda, \lambda+\delta_{1}, \lambda-\delta_{1}$ or $\lambda, \lambda-\delta_{1}, \lambda-2 \delta_{1}$ will have the properties of the first paragraph of the proof. We can assume $\lambda+\eta, \lambda+\delta_{1}+\eta, \lambda+2 \delta_{1}+\eta \notin P(V)$ for all $\eta>0$.

Note 6.5. Let $V$ be an integrable irreducible module for the twisted toroidal Lie algebra $\tau(\mu)$ with finite-dimensional weight spaces. Let $k$ be as in Theorem 2.3. Suppose $k \geq 1$ and $K_{i}=0$ for all $i$. Then such a module $V$ does not exist. This result can be proved in a way similar to the proof of [Rao 2004, Proposition 4.13].

\section{Acknowledgments}

We thank the referee for helpful comments and suggestions.

\section{References}

[Batra 2004] P. Batra, "Representations of twisted multi-loop Lie algebras", J. Algebra 272:1 (2004), 404-416. MR 2004k:17011 Zbl 1077.17005

[Berman and Billig 1999] S. Berman and Y. Billig, "Irreducible representations for toroidal Lie algebras”, J. Algebra 221:1 (1999), 188-231. MR 2000k:17004 Zbl 0942.17016

[Berman and Krylyuk 1995] S. Berman and Y. Krylyuk, "Universal central extensions of twisted and untwisted Lie algebras extended over commutative rings", J. Algebra 173:2 (1995), 302-347. MR 96e:17066 Zbl 0852.17020

[Kac 1990] V. G. Kac, Infinite-dimensional Lie algebras, 3rd ed., Cambridge University Press, 1990. MR 92k:17038 Zbl 0716.17022

[Morita and Yoshii 1985] J. Morita and Y. Yoshii, "Universal central extensions of Chevalley algebras over Laurent polynomial rings and GIM Lie algebras", Proc. Japan Acad. Ser. A Math. Sci. 61:6 (1985), 179-181. MR 87c:17028 Zbl 0581.17010

[Rao 1993] S. E. Rao, "On representations of loop algebras", Comm. Algebra 21:6 (1993), 21312153. MR 95c:17039 Zbl 0777.17019

[Rao 1995] S. E. Rao, "Iterated loop modules and a filtration for vertex representation of toroidal Lie algebras", Pacific J. Math. 171:2 (1995), 511-528. MR 97c:17034 Zbl 0867.17017

[Rao 2001] S. E. Rao, "Classification of irreducible integrable modules for multi-loop algebras with finite-dimensional weight spaces”, J. Algebra 246:1 (2001), 215-225. MR 2003c:17010 Zbl 0994.17002

[Rao 2004] S. E. Rao, "Classification of irreducible integrable modules for toroidal Lie algebras with finite dimensional weight spaces", J. Algebra 277:1 (2004), 318-348. MR 2005d:17011 Zbl 02102481

[Rao 2005] S. E. Rao, "Irreducible representations for toroidal Lie-algebras", J. Pure Appl. Algebra 202:1-3 (2005), 102-117. MR 2006e:17011 Zbl 02217995

[Rao and Moody 1994] S. E. Rao and R. V. Moody, "Vertex representations for $n$-toroidal Lie algebras and a generalization of the Virasoro algebra", Comm. Math. Phys. 159:2 (1994), 239-264. MR 94m:17028 Zbl 0808.17018 
Received November 11, 2007. Revised May 6, 2008.

S. ESWARA RAO

SCHOOL OF MATHEMATICS

TATA Institute of Fundamental RESEARCH

HOMI BHABHA ROAD

MUMBAI 400005

INDIA

senapati@math.tifr.res.in

PUNITA BATRA

HARISH CHANDRA RESEARCH INSTITUTE

CHHATNAG ROAD

JHUNSI

AllahaBAD 211019

INDIA

batra@mri.ernet.in 